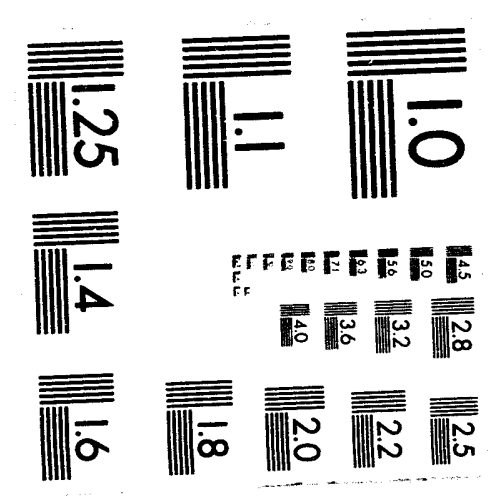



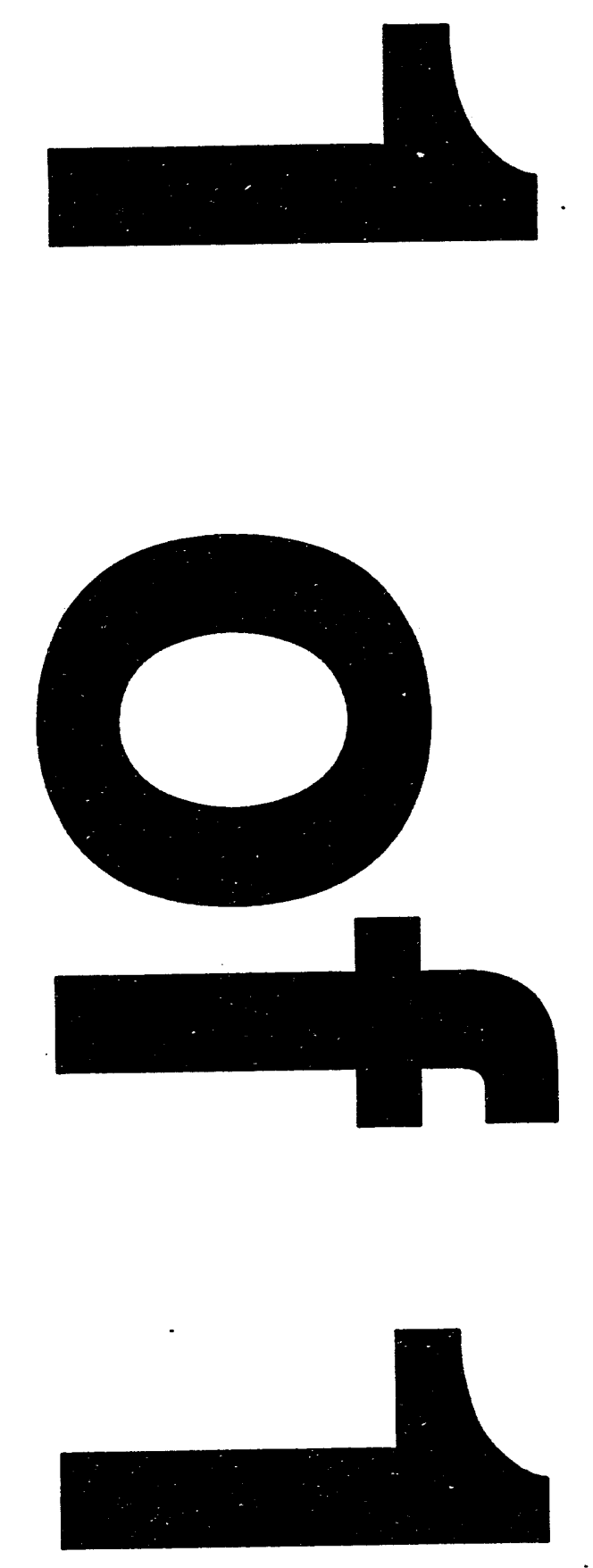


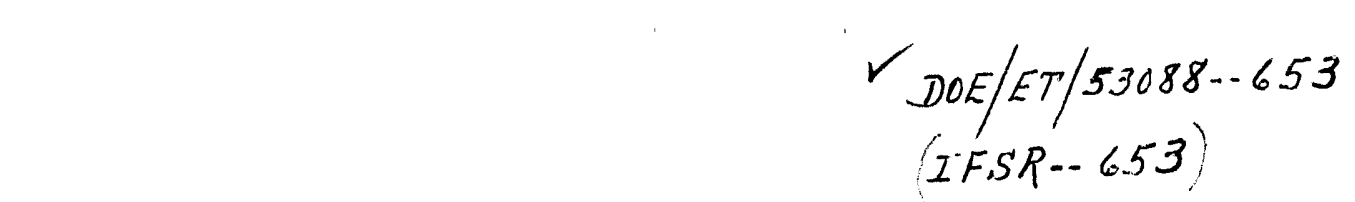

\section{INSTITUTE FOR FUSION STUDIES \\ RECEIVED
APR 11 1994 \\ OSTI}

DE-FG05-80ET-53088-653

IFSR \#653

Effect of a Static External Magnetic Perturbation on

Resistive Mode Stability in Tokamaks

RichaRd FitzPatRick AND T.C. HendeR ${ }^{a)}$

Institute for Fusion Studies

The University of Texas at Austin

Austin, Texas 78712

March 1994

${ }^{a)}$ Culham Laboratory, Abingdon, Oxon, OX14 3DB, UK
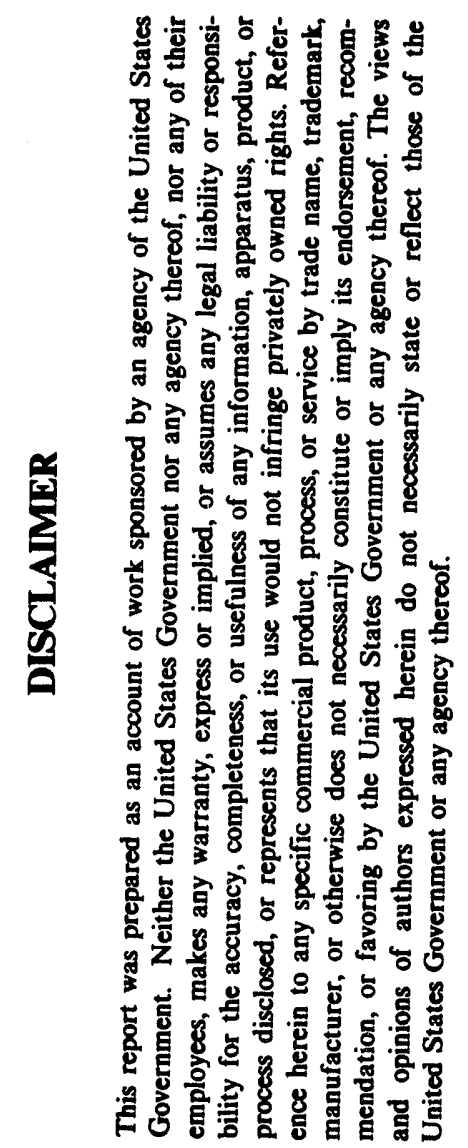

THE UNIVERSITY OF TEXAS

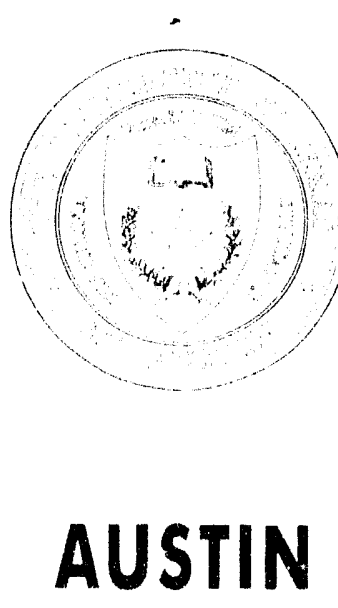

MASTER 


\title{
Effect of a Static External Magnetic Perturbation on Resistive Mode Stability in Tokamaks
}

\author{
R. FitzPATRICK AND T.C. HENDER ${ }^{a)}$ \\ Institute for Fusion Studies \\ The University of Texas at Austin \\ Austin, Texas 78712
}

\begin{abstract}
The influence of a general static external magnetic perturbation on the stability of resistive modes in a tokamak plasma is examined. There are three main parts to this investigation. Firstly, the vacuum perturbation is expanded as a set of wellbehaved toroidal ring functions and is, thereafter, specified by the coefficients of this expansion. Secondly, a dispersion relation is derived for resistive plasma instabilities in the presence of a general external perturbation and finally, this dispersion relation is solved for the amplitudes of the tearing and twisting modes driven in the plasma by a specific perturbation. It is found that the amplitudes of driven tearing and twisting modes are negligible until a certain critical perturbation strength is exceeded. Only tearing modes are driven in low- $\beta$ plasmas with $\epsilon \beta_{p} \ll 1$. However, twisting modes may also be driven if $\epsilon \beta_{p} \gtrsim 1$. For error-field perturbations made up of a large number of different poloidal and toroidal harmonics the critical strength to drive locked modes has a 'staircase' variation with edge- $q$, characterized by strong discontinuities as coupled rational surfaces enter or leave the plasma. For single harmonic perturbations

${ }^{a)}$ Culham Laboratory, Abingdon, Oxon., OX14 3DB, UK
\end{abstract}


the variation with edge- $q$ is far smoother. Both types of behaviour have been observed experimentally. The critical perturbation strength is found to decrease strongly close to an ideal external kink stability boundary. This is also in agreement with experimental observations. 


\section{Introduction}

Recent experimental and theoretical results have lead to a greatly increased understanding of the influence of a static external magnetic perturbation on resistive mode stability in a tokamak plasma. ${ }^{1-10}$ It is found that plasma rotation inhibits externally driven magnetic reconnection until a certain critical perturbation amplitude is exceeded. The critical amplitude decreases strongly with increasing machine dimensions because of the relatively slow intrinsic plasma rotation in large devices, ${ }^{9}$ but can be artificially increased by spinning the plasma with unbalanced neutral beam injection (NBI). ${ }^{5}$ The critical amplitude is sufficiently small in the largest present-day devices to allow field errors, due, for instance, to misalignment of poloidal field coils, to drive magnetic reconnection in otherwise stable plasmas. ${ }^{1,10}$ This effect can significantly reduce the disruption-free operating space at low plasma densities, and may, therefore, need to be taken into account in the ongoing International Tokamak Experimental Reactor (ITER) Engineering Design Activity. ${ }^{11}$

Existing theories describing the interaction of a static external magnetic perturbation with a rotating tokamak plasma are limited to zero- $\beta$ in slab or cylindrical geometry. ${ }^{2,6,8,9}$ We aim to extend the theory of this interaction to both finite- $\beta$ and toroidal geometry. There are, of course, a number of complications. At zero- $\beta$ only tearing parity plasma instabilities need to be taken into account, whereas at finite- $\beta$ twisting parity modes must also be included in the analysis. ${ }^{12}$ Furthermore, in toroidal geometry magnetic perturbations with different poloidal mode numbers are coupled together. As is described in Sec. II, the dispersion relation for externally driven resistive modes in a plasma possessing $N$ rational surfaces takes the form of two coupled $N \times N$ matrix equations. ${ }^{12}$ In Sec. III we use this dispersion relation to investigate the response of intrinsically stable tearing and twisting modes to a generalized external perturbation. In Secs. IV and V we classify and characterize 
the external perturbations which are likely to occur in experimental situations, using results from the recently developed T7 code. ${ }^{13}$ Our main conclusions are summarized in Sec. VI.

\section{The Dispersion Relation for Externally Driven Re- sistive Instabilities}

\section{A. Asymptotic matching}

The analysis of resistive instabilities in a high temperature tokamak is generally facilitated by dividing the plasma into two regions. ${ }^{14}$ In the 'outer' region, which comprises most of the plasma, a general instability is governed by the equations of ideal magnetohydrodynamics (MHD), which are equivalent to the requirement of force balance in an incompressible, perfectly conducting fluid. ${ }^{15}$ The 'inner' region is localized around so-called rational flux surfaces, where the helical pitch of equilibrium magnetic field lines resonates with that of the instability. The ideal MHD equations are, in fact, singular at the rational surfaces. The physical solution is obtained by asymptotically matching the outer solution across a set of thin layers centered on the rational surfaces. In these layers nonideal effects such as plasma resistivity, inertia, viscosity, and compressibility are important.

\section{B. The dispersion relation}

Suppose that there are $N$ rational surfaces in the plasma resonant with toroidal mode number $n$. Let $r_{1}<r_{2}<\cdots r_{N}$ be the minor radii of these surfaces, and $m_{1}, m_{2}, \cdots m_{N}$ the resonant poloidal mode $\mathrm{I}^{\prime}$ mbers. There are, in general, $2 N$ independent resistive modes. It is convenient to resolve a general mode into components of $N$ basis tearing modes and $N$ basis twisting modes. The $j$ th basis tearing mode $(1 \leq j \leq N)$ is defined to have unit tearing amplitude and zero twisting amplitude at rational surface $j$, with zero tearing or twisting amplitude at any other surface. Likewise, the $j$ th basis twisting mode has unit twisting amplitude and zero tearing amplitude at surface $j$, with zero tearing or twisting 
amplitude at any other surface. Here, the tearing amplitude at surface $j$ is basically the even (with respect to the rational surface) component of the perturbed normal resonant magnetic field, whereas the twisting amplitude is the odd component (see the appendix for more exact definitions).

The most general dispersion relation for coupled tearing and twisting modes in the presence of an external perturbation takes the form (see appendix) $)^{12,16}$

$$
\begin{aligned}
\left\{\boldsymbol{\Delta}^{+}(\omega)-\mathbf{E}^{+}\right\} \boldsymbol{\Psi}^{+}-\mathbf{H} \boldsymbol{\Psi}^{-}=\mathbf{C}^{+} \\
\left\{\boldsymbol{\Delta}^{-}(\omega)-\mathbf{E}^{-}\right\} \boldsymbol{\Psi}^{-}-\mathbf{H}^{\dagger} \boldsymbol{\Psi}^{+}=\mathbf{C}^{-}
\end{aligned}
$$

where $\mathbf{E}^{ \pm}$is an $N \times N$ real symmetric matrix, $\mathbf{H}$ is an $N \times N$ real matrix and $\mathbf{H}^{\dagger}$ is its transpose, $\Delta^{ \pm}(\omega)$ is the $N \times N$ complex diagonal matrix of the $\Delta_{j}^{ \pm}(\omega)$ values, $\Psi^{ \pm}$is the $1 \times N$ complex vector of the $\Psi_{j}^{ \pm}$values, and $\mathrm{C}^{ \pm}$are $1 \times N$ complex vectors characterizing the external perturbation. Here, $\Delta_{j}^{+}(\omega)$ is the tearing parity stability index for the layer at rational surface $j$, and $\Delta_{j}^{-}(\omega)$ is the corresponding twisting parity stability index [see Eq. (A.11) and Sec. II.F]. Also, $\Psi_{j}^{+}$is the tearing amplitude at surface $j$, and $\Psi_{j}^{-}$the corresponding twisting amplitude [see Eq. (A.10a)]. The tearing amplitude is sometimes termed the 'reconnected flux.'

The $\mathrm{E}^{+}$Matrix determines the intrinsic stability and mutual interaction of basis tearing modes in the plasma. The $\mathrm{E}^{-}$Matrix governs the intrinsic stability of basis twisting modes, and the $\mathrm{H}$ Matrix specifies the interaction of basis tearing and twisting modes. The evaluation of these matrices in a large aspect ratio, low- $\beta$, weakly shaped tokamak equilibrium is discussed in Refs. 12 and 13. The appendix describes how the components of the $\mathrm{C}^{ \pm}$Vectors are calculated for a given external perturbation and plasma equilibrium. 


\section{The $\mathrm{E}^{+}$matrix}

Consider a plasma with a monotonic safety factor profile containing no rational surfaces resonant with poloidal mode number $m=1$. (The restriction to $m>1$ modes is necessary because the $m=1$ mode generally requires special treatment in tokamak plasmas. ${ }^{17}$ ) In such a plasma the diagonal elements of the $\mathrm{E}^{+}$Matrix take the form

$$
E_{j j}^{+}=\Delta_{j}^{0}+\mathcal{O}\left(\epsilon^{2}\right)
$$

where $\Delta_{j}^{0}$ is the standard cylindrical tearing stability index for the $m_{j} / n$ mode (normalized with respect to $r_{j}$ ). The off-diagonal elements of the $\mathrm{E}^{+}$Matrix are $\mathcal{O}(\epsilon)$. Coupling of basis tearing modes with resonant poloidal mode numbers differing by unity is effected by the Shafranov shift of flux surfaces, which is driven by toroidicity and the plasma pressure. Coupling of modes with poloidal mode numbers differing by two or three is effected by flux surface ellipticity or triangularity, respectively. ${ }^{13}$ (The 'resonant' poloidal mode number of the $j$ th basis tearing or twisting mode is, of course, $m_{j}$.)

\section{The $\mathrm{E}^{-}$matrix}

For a plasma with a monotonic safety factor profile the $\mathrm{E}^{-}$Matrix is diagonal (for the ordering adopted in Refs. 12 and 13), indicating that there is no direct coupling of basis twisting modes possessing different resonant poloidal mode numbers. The $j$ th diagonal element can be written ${ }^{12}$

$$
E_{j j}^{-}=-\Delta_{j}^{c}+e_{j} m_{j}\left(\alpha_{j}\right)^{2},
$$

where $\Delta_{j}^{c}$ is a stabilizing term emanating from the layer at rational surface $j$, and

$$
\alpha_{j}=-\left(\frac{2 R_{0} \mu_{0} p^{\prime} q^{2}}{B_{0}^{2}}\right)_{r_{j}}
$$

is a measure of the local pressure gradient at surface $j$. Here, $R_{0}$ is the major radius of the plasma, $B_{0}$ the vacuum magnetic field strength on the magnetic axis, $r$ the minor radius 
of flux surfaces, $p(r)$ the equilibrium pressure profile, $q(r)$ the safety factor profile, and ' denotes $d / d r$. In Eq. (3), $e_{j}$ is an $\mathcal{O}(1)$ parameter which can be evaluated using $m_{j} \pm 1 / n$ cylindrical basis functions. In Ref. 12 it is demonstrated that to a good approximation

$$
\Delta_{j}^{c}=2.104 \beta_{j}^{1 / 2}\left(\frac{\tau_{R}^{2 / 3}}{\tau_{H}^{1 / 3} \tau_{V}^{1 / 3}}\right)_{r_{j}}
$$

in a typical ohmically heated tokamak plasma, where compressibility, (anomalous) viscosity, .nd resistivity are the dominant nonideal effects. In the above, $\tau_{H}=\left(R_{0} / B_{0}\right) \sqrt{\mu_{0} \rho(r)} / n s(r)$ is the hydromagnetic timescale, $\tau_{R}(r)=\mu_{0} r^{2} / \eta_{\|}(r)$ the resistive timescale, $\tau_{V}(r)=r^{2} \rho(r) / \mu_{\perp}(r)$ the viscous timescale, and $\beta_{j}=\gamma_{s} \mu_{0} p\left(r_{j}\right) / B_{0}^{2}$ is a measure of the stabilizing effect of plasma compressibility at surface $j$. Here, $\gamma_{s}$ is the standard ratio of specific heats, $\rho(r)$ the plasma mass density, $\eta_{\|}(r)$ the parallel resistivity, $\mu_{\perp}(r)$ the (anomalous) perpendicular viscosity, and $s(r)=r q^{\prime} / q$ the magnetic shear.

\section{E. The H matrix}

For a plasma with a monotonic safety factor profile the $\mathrm{H}$ Matrix is tridiagonal, indicating that basis tearing modes can couple to basis twisting modes with the same resonant poloidal mode number and with mode numbers differing by unity, and vice versa. The $j$ th diagonal element is written ${ }^{12}$

$$
H_{j j}=-\frac{\pi}{2} \frac{\kappa_{j}}{s_{j}}+\mathcal{O}\left(\epsilon^{2}\right)
$$

where

$$
\kappa_{j}=\left(-q r \frac{d}{d r}\left[\frac{1}{r} \frac{d}{d r}\left(\frac{r^{2}}{q}\right)\right]\right)_{r_{j}}+\mathcal{O}\left(\epsilon^{2}\right)
$$

is a measure of the local equilibrium current gradient at rational surface $j$. The element of the $\mathrm{H}$ Matrix which couples the basis tearing mode associated with surface $j$ to the basis twisting mode associated with surface $k$ takes the form ${ }^{12}$

$$
H_{j k}=h_{j k} m_{j} \alpha_{k}
$$


provided $m_{k}=m_{j} \pm 1$. The $\mathcal{O}(1)$ parameter $h_{j k}$ can be evaluated using $m_{j} / n$ cylindrical basis functions.

\section{F. The layer responses}

The responses of the resistive layers at the $N$ rational surfaces in the plasma to tearing and twisting parity perturbations from the outer region are specified by the diagonal matrices $\Delta^{+}$and $\boldsymbol{\Delta}^{-}$. The $j$ th diagonal element of $\boldsymbol{\Delta}^{+}$(i.e. $\Delta_{j}^{+}$) specifies the response of the $j$ th layer to a tearing parity perturbation, and the $j$ th diagonal element of $\Delta^{-}$(i.e. $\Delta_{j}^{-}$) specifies the response to a twisting parity perturbation. It turns out that the responses of resistive layers to external perturbations are resonant in nature. ${ }^{2,18}$ That is, there is virtually no tearing or twisting amplitude driven in a layer unless the external tearing or twisting parity perturbation rotates in a certain very narrow band of frequencies. The optimum frequency for externally driven tearing amplitude at surface $j$ is equal to the 'natural frequency' of the $j$ th basis tearing mode (i.e. the propagation frequency of the uncoupled, intrinsically unstable $j$ th basis tearing mode). Likewise, the optimum frequency for externally driven twisting amplitude at surface $j$ is equal to the natural frequency of the $j$ th basis twisting mode. In Ref. 12 it is shown that to a good approximation

$$
\Delta_{j}^{ \pm}(\omega)=-\mathrm{i}\left(\omega-\omega_{j}^{ \pm}\right) \tau_{j}
$$

in a typical ohmically heated tokamak plasma. Here, $\omega$ is the mode rotation frequency [all layer quantities are assumed to vary like $\exp (-\mathrm{i} \omega t)]$,

$$
\tau_{j}=2.104\left(\frac{\tau_{H}^{1 / 3} \tau_{R}^{5 / 6}}{\tau_{V}^{1 / 6}}\right)_{r_{j}}
$$

is the reconnection timescale at surface $j, \omega_{j}^{+}$is the natural frequency for tearing parity modes at this surface, and $\omega_{j}^{-}$is the corresponding natural frequency for twisting parity modes. Both natural frequencies are determined by local equilibrium plasma flows. Typically, the natural frequencies of tearing and twisting modes differ by of order the local 
electron diamagnetic frequency. ${ }^{12}$ In addition, sheared rotation and diamagnetic flows in the plasma ensure that the natural frequencies of basis modes associated with different rational surfaces are not the same.

\section{G. The $\mathbf{C}^{ \pm}$vectors}

The $\mathrm{C}^{ \pm}$Vectors are functions of both the plasma equilibrium and the external perturbation. Typical values are given in Secs. IV and V, but for the moment they are assumed to be arbitrary.

\section{Externally Driven Resistive Instabilities}

\section{A. Introduction}

In this section we aim to characterize the solutions of the dispersion relation (1), as fully as possible, for the case of an arbitrary static external magnetic perturbation interacting with an intrinsically stable plasma. Section III.B examines the simple case where there is only one rational surface in the plasma. Section III.C describes the more complicated situation where there are two rational surfaces, and Sec. III.D briefly examines the case of three (or more) surfaces.

\section{B. Stability of a plasma containing a single rational surface}

\section{Introduction}

Consider the simplest possible situation where there is only a single rational surface in the plasma, radius $r_{1}$, resonant with poloidal mode number $m_{1}$. In this case the dispersion relation (1) reduces to

$$
\Psi_{1}^{+}=\frac{\left(\Delta_{1}^{-}-E_{11}^{-}\right) C_{1}^{+}+H_{11} C_{1}^{-}}{\left(\Delta_{1}^{+}-E_{11}^{+}\right)\left(\Delta_{1}^{-}-E_{11}^{-}\right)-\left(H_{11}\right)^{2}}
$$




$$
\Psi_{1}^{-}=\frac{\left(\Delta_{1}^{+}-E_{11}^{+}\right) C_{1}^{-}+H_{11} C_{1}^{+}}{\left(\Delta_{1}^{+}-E_{11}^{+}\right)\left(\Delta_{1}^{-}-E_{11}^{-}\right)-\left(H_{11}\right)^{2}} .
$$

Now, $\left|\omega_{1}^{+}-\omega_{1}^{-}\right| \tau_{1} \gg 1$ in a typical high temperature tokamak plasma, ${ }^{12,18}$ where $\omega_{1}^{+}$is the natural frequency of tearing parity modes at the rational surface, $\omega_{1}^{-}$is the natural frequency of twisting parity modes, and $\tau_{1}$ is the reconnection timescale. It follows from Eqs. (9) that $\Delta_{1}^{+}$and $\Delta_{1}^{-}$are never small simultaneously, so Eqs. (11) reduce to

$$
\begin{aligned}
& \Psi_{1}^{+} \simeq \frac{C_{1}^{+}}{\mathrm{i} \omega_{1}^{+} \tau_{1}-E_{11}^{+}}, \\
& \Psi_{1}^{-} \simeq \frac{C_{1}^{-}}{\mathrm{i} \omega_{1}^{-} \tau_{1}-E_{11}^{-}},
\end{aligned}
$$

assuming that the perturbation is static (i.e. $\omega=0$ ). It is also assumed that the tearing and twisting modes are both intrinsically stable, so that $E_{11}^{+}<0$ and $E_{11}^{-}<0$. It is clear from (12) that the $\mathrm{C}^{+}$Vector drives tearing parity modes in the plasma, whereas the $\mathrm{C}^{-}$Vector drives twisting parity modes.

\section{Electromagnetic and viscous torques}

The nonlinear toroidal electromagnetic torque acting at rational surface 1 is given by

$$
\begin{aligned}
\delta T_{\phi \mathrm{EM}}\left(r_{1}\right)= & \frac{2 n \pi^{2} R_{0}}{\mu_{0}} \times \\
& {\left[\frac{\omega_{1}^{+} \tau_{1}}{\left(\omega_{1}^{+} \tau_{1}\right)^{2}+\left(-E_{11}^{+}\right)^{2}}\left|C_{1}^{+}\right|^{2}+\frac{\omega_{1}^{-} \tau_{1}}{\left(\omega_{1}^{-} \tau_{1}\right)^{2}+\left(-E_{11}^{-}\right)^{2}}\left|C_{1}^{-}\right|^{2}\right], }
\end{aligned}
$$

where use has been made of Eqs. (9), (12), and (A.12). This torque modifies the bulk toroidal rotation..$^{9,12,19}$ (It is assumed that any modifications to the bulk poloidal rotation are prevented by strong poloidal flow damping.) The steady-state shift induced in the plasma toroidal angular rotation velocity is ${ }^{9,19}$

$$
\Omega_{\phi}(r)=\Omega_{\phi}\left(r_{1}\right) \begin{cases}1 & r<r_{1} \\ \int_{r}^{a} \frac{d r}{r \mu_{\perp}(r)} / \int_{r_{1}}^{a} \frac{d r}{r \mu_{\perp}(r)} & r_{1} \leq r \leq a,\end{cases}
$$


where $a$ is the minor radius of the outermost plasma flux surface. Here, it is assumed that the toroidal rotation is 'clamped' at the edge $(r=a)$, so that $\Omega_{\phi}(a)=0.5$ The viscous restoring torque which develops at the rational surface is given by ${ }^{9,19}$

$$
\begin{aligned}
\delta T_{\phi \mathrm{VS}}\left(r_{1}\right) & =4 \pi^{2} R_{0} \times\left[\left(r \mu_{\perp} R_{0}^{2}\right) \frac{d \Omega_{\phi}}{d r}\right]_{r_{1-}}^{r_{1+}} \\
& =-4 \pi^{2} R_{0} \times \Omega_{\phi}\left(r_{1}\right) R_{0}^{2} \times / \int_{r_{1}}^{a} \frac{d r}{r \mu_{\perp}(r)} .
\end{aligned}
$$

In a steady-state plasma the viscous and electromagnetic torques must balance, so

$$
\delta T_{\phi \mathrm{EM}}\left(r_{1}\right)+\delta T_{\phi \mathrm{VS}}\left(r_{1}\right)=0
$$

Finally, the changes induced in the plasma toroidal angular velocity profile Doppler shift the various natural frequencies, so that

$$
\omega_{1}^{ \pm} \rightarrow \omega_{1}^{ \pm}-n \Omega_{\phi}\left(r_{1}\right),
$$

where $\omega_{1}^{ \pm}$now denote natural frequencies in the unperturbed plasma.

\section{Torque balance}

The balance of electromagnetic and viscous torques in the plasma yields

$$
\frac{1}{4} \frac{y^{+}(1-f)}{\left(b^{+}\right)^{2}+(1-f)^{2}}-\frac{1}{4} \frac{y^{-} f}{\left(b^{-}\right)^{2}+f^{2}}=f-f_{1},
$$

where

$$
\begin{aligned}
f & =\frac{n \Omega_{\phi}\left(r_{1}\right)-\omega_{1}^{-}}{\omega_{1}^{+}-\omega_{1}^{-}}, \\
f_{1} & =\frac{-\omega_{1}^{-}}{\omega_{1}^{+}-\omega_{1}^{-}}, \\
b^{ \pm} & =\frac{-E_{11}^{ \pm}}{\left|\omega_{1}^{+}-\omega_{1}^{-}\right| \tau_{1}},
\end{aligned}
$$




$$
\begin{aligned}
& y^{ \pm}=\frac{\left|C_{1}^{ \pm}\right|^{2}}{\Lambda^{2}} \\
& \Lambda^{2}=\frac{\mu_{0}}{2} \frac{R_{0}^{2}}{n^{2}}\left(\omega_{1}^{+}-\omega_{1}^{-}\right)^{2} \tau_{1} / \int_{r_{1}}^{a} \frac{d r}{r \mu_{\perp}(r)} .
\end{aligned}
$$

In the physically relevant asymptotic limit $b^{ \pm} \ll 1,{ }^{12}$ Eq. (18) possesses bifurcated solutions. Bifurcations occur in the $y^{+}-y^{-}$plane when the curve of lccus

$$
\begin{aligned}
& y^{+}=4\left(2 f-f_{1}\right)(1-f)^{2}, \\
& y^{-}=4\left(1-2 f+f_{1}\right) f^{2}
\end{aligned}
$$

is crossed in the direction of increasing $y^{+}$and $y^{-}$. This critical curve is plotted in Fig. 1. The solution can either bifurcate to the tearing resonance at surface $1(f=1)$, or the associated twisting resonance $(f=0)$. Prior to bifurcation there is very little driven tearing or twisting amplitude, whereas after bifurcation, or 'locking', to the tearing resonance there is substantial driven tearing amplitude at surface 1 ,

$$
\Psi_{1}^{+} \simeq \frac{C_{1}^{+}}{\left(-E_{11}^{+}\right)}
$$

and likewise after locking to the twisting resonance there is substantial driven twisting amplitude,

$$
\Psi_{1}^{-} \simeq \frac{C_{1}^{-}}{\left(-E_{11}^{-}\right)} .
$$

Locking to either resonance is associated with a sudden change in the steady-state plasma rotation such as to bring the Doppler shifted natural frequency of the tearing or twisting mode at surface 1 (as appropriate) into coincidence with the applied frequency (which is, of course, zero for a static external perturbation).

Suppose that $\left|C_{1}^{+}\right|^{2} \gg\left|C_{1}^{-}\right|^{2}$ (i.e. the locking torque exerted at the rational surface due to externally driven tearing amplitude is much greater than that due to driven twisting 
amplitude). As is described in Sec. $V$, this is likely to be the case in low- $\beta$ tokamak plasmas. In this limit, locking to the tearing resonance takes place when the Doppler shifted natural frequency for tearing modes is reduced to one half of its original value [i.e. $\omega_{1}^{+}-n \Omega_{\phi}\left(r_{1}\right)=$ $\left.\frac{1}{2} \omega_{1}^{+}\right]$. This takes place when

$$
\left|C_{1}^{+}\right|^{2} \geq \Lambda_{1}^{2} \equiv \frac{\mu_{0}}{2} \frac{R_{0}^{2}}{n}\left(\omega_{1}^{+}\right)^{2} \tau_{1} / \int_{r_{1}}^{a} \frac{d r}{r \mu_{\perp}(r)} .
$$

\section{Discussion}

It is clear that the tearing and twisting amplitude driven in a stable tokamak plasma by a static external magnetic perturbation is a highly nonlinear function of the perturbation strength. In fact, there is virtually no driven amplitude until the perturbation strength exceeds a critical value (i.e. $\left|C_{1}^{+}\right|,\left|C_{1}^{-}\right| \sim \Lambda$ ), at which point substantial tearing or twisting amplitude is driven in the plasma. Externally driven tearing amplitude at surface 1 leads to the formation of a stationary chain of magnetic islands whose width is proportional to the square root of the amplitude. ${ }^{20}$ Externally driven twisting amplitude leads to the formation of a much narrower chain of 'skewed' magnetic islands whose width is directly proportional to the amplitude. Thus, locking of the external perturbation to the twisting resonance at surface 1 is likely to cause less degradation of the plasma confinement than locking to the tearing resonance. A threshold effect for externally induced magnetic tearing in tokamaks has been observed experimentally. $1,3,10$

\section{Stability of a plasma containing two rational surfaces}

\section{Introduction}

Suppose that there are two rational surfaces in the plasma (labelled 1 and 2, with $r_{2}>r_{1}$ ). It is assumed that the basis tearing and twisting modes associated with the two surfaces are all intrinsically stable, so that $E_{11}^{ \pm}<0$ and $E_{22}^{ \pm}<0$. In the following, our investigation 
is limited to tearing parity modes, for the sake of simplicity. In fact, as is discussed in Section $\mathrm{V}$, the components of the $\mathrm{C}^{-}$Vector, which drive twisting parity modes in the plasma, are proportional to the plasma pressure and can, therefore, be neglected in low- $\beta$ devices.

\section{Electromagnetic and viscous torques}

In the physically relevant limit, $\omega_{j}^{+} \tau_{j} \gg 1$ (where $j$ is 1 or 2 ), the tearing amplitudes driven by a general static external perturbation are given by

$$
\Psi_{j}^{+} \simeq-\mathrm{i} \frac{C_{j}^{+}}{\omega_{j}^{+} \tau_{j}},
$$

and the localized electromagnetic locking torques exerted at the rational surfaces are written

$$
\delta T_{\phi \mathrm{EM}}\left(r_{j}\right) \simeq \frac{2 n \pi^{2} R_{0}}{\mu_{0}} \times \frac{\left|C_{j}^{+}\right|^{2}}{\omega_{j}^{+} \tau_{j}} .
$$

The steady-state shift induced in the plasma toroidal angular velocity profile by these torques takes the form

$$
\Omega_{\phi}(r)= \begin{cases}\Omega_{\phi}\left(r_{1}\right) & r<r_{1} \\ \Omega_{\phi}\left(r_{2}\right)+\left[\Omega_{\phi}\left(r_{1}\right)-\Omega_{\phi}\left(r_{2}\right)\right] \int_{r}^{r_{2}} \frac{d r}{r \mu_{\perp}(r)} / \int_{r_{1}}^{r_{2}} \frac{d r}{r \mu_{\perp}(r)} & r_{1} \leq r \leq r_{2} \\ \Omega_{\phi}\left(r_{2}\right) \int_{r}^{a} \frac{d r}{r \mu_{\perp}(r)} / \int_{r_{2}}^{a} \frac{d r}{r \mu_{\perp}(r)} & r_{2}<r \leq a,\end{cases}
$$

which implies the following localized viscous torques acting inside the plasma:

$$
\begin{aligned}
& \delta T_{\phi \mathrm{VS}}\left(r_{1}\right)=-4 \pi^{2} R_{0} \times\left[\Omega_{\phi}\left(r_{1}\right)-\Omega_{\phi}\left(r_{2}\right)\right] R_{0}^{2} / \int_{r_{1}}^{r_{2}} \frac{d r}{r \mu_{\perp}(r)} \\
& \delta T_{\phi \mathrm{VS}}\left(r_{2}\right)=-\delta T_{\phi \mathrm{VS}}\left(r_{1}\right)-4 \pi^{2} R_{0} \times \Omega_{\phi}\left(r_{2}\right) R_{0}^{2} / \int_{r_{2}}^{a} \frac{d r}{r \mu_{\perp}(r)} .
\end{aligned}
$$

As before, the changes induced in the plasma rotation Doppler shift the various natural frequencies, so that

$$
\omega_{j}^{+} \rightarrow \omega_{j}^{+}-n \Omega_{\phi}\left(r_{j}\right)
$$




\section{Torque balance}

After some manipulation, torque balance at the two rational surfaces yields the following pair of coupled quadratic equations:

$$
\begin{aligned}
& x^{2}-\left(1-\frac{1}{4} \kappa \frac{\beta^{2}}{y}\right) x+\frac{1}{4} \alpha^{2}=0, \\
& y^{2}-\left(1-\frac{1}{4} \frac{\lambda}{\kappa} \frac{\alpha^{2}}{x}\right) y+\frac{1}{4} \beta^{2}=0,
\end{aligned}
$$

where the variables

$$
x=\frac{\omega_{1}^{+}-n \Omega_{\phi}\left(r_{1}\right)}{\omega_{1}^{+}}, \quad y=\frac{\omega_{2}^{+}-n \Omega_{\phi}\left(r_{2}\right)}{\omega_{2}^{+}}
$$

are the Doppler shifted natural frequencies normalized with respect to their unperturbed values. The components of the external magnetic perturbation are specified by the parameters

$$
\alpha=\left|\frac{C_{1}^{+}}{\Lambda_{1}}\right|, \quad \beta=\left|\frac{C_{2}^{+}}{\Lambda_{2}}\right|
$$

where

$$
\Lambda_{j}^{2}=\frac{\mu_{0}}{2} \frac{R_{0}^{2}}{n^{2}}\left(\omega_{j}^{+}\right)^{2} \tau_{j} / \int_{r_{j}}^{a} \frac{d r}{r \mu_{\perp}(r)} .
$$

Note that $\alpha=1$ corresponds to the critical external perturbation strength required for locking to the tearing resonance at surface 1 in the absence of any torque exerted on surface 2 , and vice versa. The two remaining parameters,

$$
\begin{aligned}
& \kappa=\frac{\omega_{2}^{+}}{\omega_{1}^{+}} \\
& \lambda=\int_{r_{2}}^{a} \frac{d r}{r \mu_{\perp}(r)} / \int_{r_{1}}^{a} \frac{d r}{r \mu_{\perp}(r)},
\end{aligned}
$$

depend on the nature of the unperturbed plasma equilibrium. Note that $0 \leq \lambda<1$, since $r_{2}>r_{1}$ and $\mu_{\perp}(r)>0$. 


\section{Locking at surface 1}

According to Eqs. (29), locking to the tearing resonance at the innermost rational surface (i.e. surface 1) occurs when $\alpha \geq \alpha_{\text {crit }}$, where

$$
\alpha_{\text {crit }}=1-\frac{\kappa}{\left(1-\frac{1}{2} \lambda\right)}\left\{\frac{1}{2}\left(1-\frac{1}{2} \frac{\lambda}{\kappa}\right)-\frac{1}{2} \sqrt{\left(1-\frac{1}{2} \frac{\lambda}{\kappa}\right)^{2}-\beta^{2}\left(1-\frac{1}{2} \lambda\right)}\right\} .
$$

The normalized Doppler shifted natural frequencies just prior to locking are given by $x_{\text {crit }}=$ $\frac{1}{2} \alpha_{\text {crit }}$ and

$$
y_{\text {crit }}=\frac{1}{2}\left(1-\frac{1}{2} \frac{\lambda}{\kappa}\right)+\frac{1}{2} \sqrt{\left(1-\frac{1}{2} \frac{\lambda}{\kappa}\right)^{2}-\beta^{2}\left(1-\frac{1}{2} \lambda\right)} .
$$

Locking to the tearing resonance at the outermost rational surface (i.e. surface 2) occurs when $\beta>\beta_{\text {crit }}$, where

$$
\beta_{\text {crit }}=1-\frac{\lambda}{\kappa\left(1-\frac{1}{2} \lambda\right)}\left\{\frac{1}{2}\left(1-\frac{1}{2} \kappa\right)-\frac{1}{2} \sqrt{\left(1-\frac{1}{2} \kappa\right)^{2}-\alpha^{2}\left(1-\frac{1}{2} \lambda\right)}\right\}
$$

and

$$
x_{\text {crit }}=\frac{1}{2}\left(1-\frac{1}{2} \kappa\right)+\frac{1}{2} \sqrt{\left(1-\frac{1}{2} \kappa\right)^{2}-\alpha^{2}\left(1-\frac{1}{2} \lambda\right)}
$$

with $y_{\text {crit }}=\frac{1}{2} \beta_{\text {crit. }}$. Finally, simultaneous locking to the tearing resonances at both surfaces occurs when $\alpha=\alpha_{0}$ and $\beta=\beta_{0}$, where

$$
\alpha_{0}=\frac{\left(1-\frac{1}{2} \kappa\right)}{\left(1-\frac{1}{4} \lambda\right)}, \quad \beta_{0}=\frac{\left(1-\frac{1}{2} \frac{\lambda}{\kappa}\right)}{\left(1-\frac{1}{4} \lambda\right)}
$$

The normalized Doppler shifted natural frequencies just before simultaneous locking are $x_{0}=\frac{1}{2} \alpha_{0}$ and $y_{0}=\frac{1}{2} \beta_{0}$.

Note that if $\beta=0$ (i.e. if the external perturbation exerts no torque at surface 2 ) then locking to the tearing resonance at surface 1 occurs when $\alpha=1$ (i.e. $\left|C_{1}^{+}\right|=\Lambda_{1}$ ) with $x=\frac{1}{2}$ [i.e. $\omega_{1}-n \Omega_{\phi}\left(r_{1}\right)=\frac{1}{2} \omega_{1}$ ], which is equivalent to the simple cylindrical result of Sec. III.B (neglecting twisting modes). However, if $\beta>0$ then locking to the tearing resonance at surface 1 occurs when $\alpha<1$ (i.e. the threshold locking torque at surface 1 is reduced) with 
$x<\frac{1}{2}$ [i.e. $\omega_{1}-n \Omega_{\phi}\left(r_{1}\right)<\frac{1}{2} \omega_{1}$ ]. Similarly, if $\alpha \neq 0$ then both the threshold torque required to lock the tearing resonance at surface 2 and the critical Doppler shifted natural frequency just prior to locking are less than the cylindrical values.

Figures 2(a)-(e) show the locking thresholds for the tearing resonance calculated from Eqs. (29) for five different values of the parameter $\kappa$, which measures the ratio of the unperturbed natural frequencies for tearing parity modes at the two rational surfaces [see Eq. (33a)]. The thresholds are plotted at constant $\lambda$ in $\alpha^{2}-\beta^{2}$ space. The locus of the point of simultaneous locking of both surfaces is also plotted. The parameter $\lambda$ is strongly dependent on the relative positions of the two rational surfaces: $\lambda \rightarrow 1$ as the two surfaces approach one another, and $\lambda \rightarrow 0$ as the outermost surface app:oaches the edge of the plasma [see Eq. (33b)]. The variables $\alpha^{2}$ and $\beta^{2}$ are proportional to the locking torques exerted at surfaces 1 and 2, respectively [see Eqs. (25) and (31)]. These torques are normalized with respect to the threshold locking torques for each surface taken in isolation. For example, $\alpha=1$ corresponds to the threshold torque required for locking to the tearing resonance at surface 1 when no torque is exerted on surface 2. In Figs. 2, locking does not take place in the region of parameter space bounded by the threshold curve and the lines $\alpha=0$ and $3=0$. If the threshold curve is crossed (e.g. by increasing the amplitude of the external perturbation) then locking of either surface 1 or 2 occurs. Surface 2 is locked if the threshold curve is crossed on the low- $\alpha$ side of the simultaneous locking curve, whereas surface 1 is locked if the threshold curve is crossed on the high- $\alpha$ side.

It can be seen from Figs. 2 that reducing the parameter $\kappa$ favors the locking of surface 2. This is not surprising, since reducing $\kappa$ corresponds to reducing the unperturbed natural frequency of tearing modes at surface 2 with respect to that at surface 1 . For $\kappa<\frac{1}{2}$ and $\lambda<2 \kappa$, surface 2 always locks before surface 1 . Similarly, for $\kappa>2$, surface 1 always locks before surface 2 . Increasing $\lambda$ tends to decrease the threshold torques required to induce locking. This is also not surprising, since if $\lambda \simeq 0$ (i.e. if surface 2 lies very close to the plasma 
edge) a velocity change induced at surface 1 has little effect at surface 2 [see Eq. (26)], so the surface 2 locking criterion is virtually unaffected by the presence of surface 1 . On the other hand, a velocity change induced at surface 2 always has an effect at surface 1 , so the locking threshold for surface 1 is reduced somewhat by the presence of surface 2 . This type of mutual interaction between the two surfaces is strongest when they are very close together (i.e. when $\lambda \rightarrow 1$ ) so the locking threshold is naturally smallest in this case.

\section{Locking at surface 2}

Suppose that the tearing resonance at the outermost surface (i.e. surface 2) is locked, so that $n \Omega_{\phi}\left(r_{2}\right)=\omega_{2}^{+}$, but that the innermost surface (i.e. surface 1) remains unlocked. It is easily demonstrated that in the physically relevant limit the tearing amplitude driven at surface 1 satisfies

$$
\Psi_{1}^{+} \simeq-\mathrm{i} \frac{\tilde{C}_{1}^{+}}{\left[\omega_{1}^{+}-n \Omega_{\phi}\left(r_{1}\right)\right] \tau_{1}},
$$

where

$$
\tilde{C}_{1}^{+}=C_{1}^{+}+\frac{E_{12}^{+}}{\left(-E_{22}^{+}\right)} C_{2}^{+} .
$$

The usual analysis reveals that locking to the tearing resonance at surface 1 occurs when

$$
\left|\tilde{C}_{1}^{+}\right| \geq \frac{|1-\kappa|}{\sqrt{1-\lambda}} \Lambda_{1} .
$$

If the tearing resonance at surface 1 is locked but surface 2 remains unlocked then the tearing amplitude driven at surface 2 satisfies

$$
\Psi_{2}^{+} \simeq-\mathrm{i} \frac{\tilde{C}_{2}^{+}}{\left[\omega_{2}^{+}-n \Omega_{\phi}\left(r_{2}\right)\right] \tau_{2}},
$$

where

$$
\tilde{C}_{2}^{+}=C_{2}^{+}+\frac{E_{12}^{+}}{\left(-E_{11}^{+}\right)} C_{1}^{+} .
$$


It is easily demonstrated that in this case locking to the tearing resonance at surface 2 occurs when

$$
\left|\tilde{C}_{2}^{+}\right| \geq \frac{\left|1-\frac{\lambda}{\kappa}\right|}{\sqrt{1-\lambda}} \Lambda_{2}
$$

\section{Discussion}

It is clear from the above analysis that the situation with two rational surfaces in the plasma is far more complicated than that with only one surface, even when twisting resonances are neglected. In general, locking to the tearing resonance at a given rational surface is facilitated by the electromagnetic torque exerted at the other surface, so the locking threshold is reduced somewhat below the single surface value. The critical Doppler shifted natural frequency just before locking is also generally less than the single surface value.

\section{Stability of a plasma containing three (or more) rational sur- faces}

Suppose that there are three rational surfaces in the plasma (labelled 1, 2, and 3, with $\left.r_{3}>r_{2}>r_{1}\right)$. It is assumed that all of the basis plasma modes are intrinsically stable, and the twisting resonances are again neglected.

Application of the previous analysis to this case, in the physically relevant limit $\omega_{i}^{+} \tau_{j} \gg 1$, yields the following set of coupled quadratic equations which control locking to the tearing resonances at the three rational surfaces:

$$
\begin{aligned}
& x^{2}-\left(1-\frac{1}{4} \kappa_{21} \frac{\beta^{2}}{y}-\frac{1}{4} \kappa_{31} \frac{\gamma^{2}}{z}\right) x+\frac{1}{4} \alpha^{2}=0, \\
& y^{2}-\left(1-\frac{1}{4} \frac{\lambda_{21}}{\kappa_{21}} \frac{\alpha^{2}}{x}-\frac{1}{4} \kappa_{32} \frac{\gamma^{2}}{z}\right) y+\frac{1}{4} \beta^{2}=0, \\
& z^{2}-\left(1-\frac{1}{4} \frac{\lambda_{31}}{\kappa_{31}} \frac{\alpha^{2}}{x}-\frac{1}{4} \frac{\lambda_{32}}{\kappa_{32}} \frac{\beta^{2}}{y}\right) z+\frac{1}{4} \gamma^{2}=0
\end{aligned}
$$


The additional variable $z=\left[\omega_{3}^{+}-n \Omega_{\phi}\left(r_{3}\right)\right] / \omega_{3}^{+}$is the normalized Doppler shifted natural frequency at rational surface 3. The parameter $\gamma=\left|C_{3}^{+} / \Lambda_{3}\right|$ specifies the locking torque at surface 3: $\gamma=1$ corresponds to the torque needed for locking to the tearing resonance at this surface when no torques are applied at surfaces 1 and 2. Finally, the parameters

$$
\begin{aligned}
\kappa_{i j} & =\frac{\omega_{i}^{+}}{\omega_{j}^{+}} \\
\lambda_{i j} & =\int_{r_{i}}^{a} \frac{d r}{r \mu_{\perp}(r)} / \int_{r_{j}}^{a} \frac{d r}{r \mu_{\perp}(r)}
\end{aligned}
$$

depend on the nature of the unperturbed plasma equilibrium.

Equations (45) can be solved in much the same manner as Eqs. (29) to give the locking thresholds for the various rational surfaces. It is also fairly clear how to extend Eqs. (45) to describe the situation where there are an arbitrary number of surfaces in the plasma.

\section{E. Summary}

The above analysis is clearly far more complicated than the zero- $\beta$, cylindrical analysis of Refs. 9 and 19. There are two main reasons for this. Firstly, at finite- $\beta$ there is a twisting resonance, as well as a tearing resonance, at every rational surface in the plasma, to which the external perturbation can lock (see Sec. III.B). Secondly, the coupling of different poloidal harmonics in toroidal geometry ensures that even a single helicity external perturbation exerts electromagnetic torques simultaneously at more than one rational surface in the plasma (see Secs. III.C and III.D). In the above, these effects are investigated separately, but the analysis can easily be extended to deal with both effects simultaneously. 


\section{Calculation of the $\mathrm{C}^{+}$vector}

\section{A. Introduction}

The aim of this section is to classify and characterize the $\mathrm{C}^{+}$Vectors which are likely to occur in experimental situations. It is demonstrated in the appendix that the $\mathrm{C}^{+}$Vector is a function of both the plasma equilibrium and the external perturbation, so in Secs. IV.B and IV.C we describe how these are specified in our investigation. Section IV.D describes the $\mathrm{C}^{+}$Vector associated with a narrow spectrum external perturbation, whereas Sec. IV.E deals with the $\mathrm{C}^{+}$Vector from a broad spectrum perturbation.

\section{B. Specification of the plasma equilibrium}

Consider an equilibrium in which the locus of the flux surfaces is given by

$$
\begin{aligned}
& R=R_{0}-r \cos \omega-\Delta(r)+E(r) \cos \omega+T(r) \cos 2 \omega+\mathcal{O}\left(\epsilon^{2} a\right) \\
& Z=r \sin \omega+E(r) \sin \omega+T(r) \sin 2 \omega+\mathcal{O}\left(\epsilon^{2} a\right)
\end{aligned}
$$

Here, $(R, \phi, Z)$ are standard cylindrical polar coordinates (with $Z$ in the direction of the toroidal symmetry axis), $R_{0}$ is the plasma major radius, $r$ is a radius-like flux surface label, $\omega$ is the poloidal angle about the magnetic axis $(r=0), \Delta(r)$ is the Shafranov shift of flux surfaces, $E(r)$ is the flux surface ellipticity, and $T(r)$ is the flux surface triangularity.

The outermost plasma flux surface lies at $r=a$, where $a$ is the plasma minor radius. The ordering assumptions are that $\epsilon \equiv a / R_{0} \ll 1$, and $\Delta(a), E(a), T(a) \sim \mathcal{O}(\epsilon a)$.

The safety factor profile $q(r)$ is assumed to satisfy

$$
q(r)=q_{a} \frac{(r / a)^{2}}{1-\left[1-(r / a)^{2}\right]^{q_{a} / q_{0}}}-q_{a} \frac{\lambda}{2}(r / a)^{2 k}\left[1-(r / a)^{2}\right]
$$

where $q_{0}$ is the central safety factor, $q_{a}$ is the edge safety factor, and $k$ is a positive integer. The $\mathcal{O}\left(\epsilon^{2}\right)$ edge shear parameter $\lambda$ is chosen so that the plasma current is zero at $r=a \cdot{ }^{13}$ In 
the cylindrical limit, Eq. (48) corresponds to an equilibrium toroidal current profile of the form $j(r)=j_{0}\left[1-(r / a)^{2}\right]^{q_{a} / q_{0}-1}$.

The plasma pressure profile is assumed to satisfy

$$
p=p_{0}\left[1-(r / a)^{2}\right]^{2}
$$

Here, $p_{0}$ is the central plasma pressure, which is conveniently parameterized by the (cylindrical) poloidal beta

$$
\beta_{p}=4 \mu_{0}\left(\frac{q_{a}}{\epsilon a B_{0}}\right)^{2} \int_{0}^{a} r p(r) d r .
$$

The adopted ordering scheme requires $\epsilon \beta_{p}$ to be small compared with unity.

\section{Specification of the external magnetic perturbation}

A static external magnetic perturbation can be generated via helical windings or saddle coils, but can also arise by accident if the poloidal and toroidal field coils which support the plasma equilibrium are not properly aligned. An accidentally induced magnetic perturbation is usually referred to as an 'error field.' A general external perturbation (with a given toroidal mode number $n$ ) can be completely specified by a set of complex amplitudes, the $I_{m}$, which are basically the expansion coefficients of the vacuum magnetic scalar potential interior to the generating coils in the well behaved (as $r \rightarrow 0$ ) toroidal ring functions [see Eqs. (A.18), (A.34), (A.39), and (A.40)]. The vacuum perturbation within the generating coils can be shown to reduce to

$$
\left(\delta B_{r}\right)_{\mathrm{cyl}} \simeq \mathrm{i} \sum_{m \neq 0} \frac{I_{m}}{a}\left(\frac{r}{a}\right)^{|m|-1} \exp [\mathrm{i}(m \theta-n \phi)]
$$

in the cylindrical limit [see Eqs. (A.4a), (A.19), (A.21), and (A.22)]. Here, $\theta$ is a 'straight' poloidal angle which is defined to be zero on the inboard mid-plane. ${ }^{13}$

Table I shows the $I_{m}$ for a typical $n=1$ external perturbation generated by saddle coils. This example field was employed during a series of controlled experiments performed on 
the COMPASS-D tokamak and was designed to have predominantly $m / n=2 / 1$ helicity. ${ }^{21}$ Hence, $I_{2}$ is the dominant amplitude after $I_{0}(m=0$ perturbations do not interact with resistive modes - see Sec. IV.E).

\begin{tabular}{|r||lr|}
\hline$m$ & $\left|I_{m}\right| \phi_{m}\left(^{\circ}\right)$ \\
\hline-1 & 0.11 & 180 \\
0 & 2.52 & 180 \\
1 & 0.11 & 180 \\
2 & 1.00 & 0 \\
3 & 0.34 & 180 \\
4 & 0.19 & 180 \\
5 & 0.26 & 0 \\
\hline
\end{tabular}

Table I Relative amplitudes and phases of the $I_{m}$ for a typical $n=1$ CoMPASS-D RMP field generated by external saddle coils, with $1 \mathrm{kA}$ in the saddle bars, $R_{0}=0.56 \mathrm{~m}$ and $a=0.20 \mathrm{~m}$. The absolute amplitude of $I_{2}$ is $8.6 \times 10^{-5} \mathrm{Tm}$.

Table II shows the $I_{m}$ for a typical error field. This example field was generated by the Joint European Torus (JET) poloidal field coil set for a standard limiter discharge with $q_{a} \simeq 3.2 .{ }^{10}$ It exhibits the broad spectrum which is characteristic of an error field. Note that all of the even- $m$ amplitudes are approximately $180^{\circ}$ out of phase with the odd- $m$ amplitudes, indicating a dipole like error field source localized close to the outboard mid-plane $(\theta=\pi)$. In fact, it is known that the dominant contribution to the JET error field in this discharge comes from a pair of vertical field coils (usually referred to as P4) located just above and below the outboard mid-plane. ${ }^{10}$

In the following, we investigate the effect of two idealized $n=1$ static magnetic perturbations (representative of those in Tables I and II) on the stability of the $2 / 1$ tearing mode. The first perturbation is supposed to be representative of the type generally used in 'resonant magnetic perturbation' (RMP) experiments, ${ }^{3,21}$ and has

$$
I_{2} \neq 0, \quad I_{m \neq 2}=0 .
$$

The second perturbation is supposed to represent an error field generated by a source located 


\begin{tabular}{|r|lr|}
\hline$m$ & $\left|I_{m}\right|$ & $\phi_{m}\left({ }^{\circ}\right)$ \\
\hline-1 & $0.39-178.3$ \\
0 & 1.43 & +5.0 \\
1 & $1.29-179.1$ \\
2 & 1.00 & 0.0 \\
3 & $0.65+178.4$ \\
4 & 0.35 & -4.4 \\
5 & $0.14+169.0$ \\
\hline
\end{tabular}

Table II Relative amplitudes and phases of the $I_{m}$ for the $n=1$ error field generated by the JET poloidal field coil set for a typical limiter discharge with $q_{a} \sim 3.2$. Here, the plasma current and toroidal field are in the same direction, there are 39 turns in the P4 coils, $R_{0}=2.96 \mathrm{~m}$, and $a=1.0 \mathrm{~m}$. The absolute amplitude of $I_{2}$ is $2.6 \times 10^{-4} \mathrm{~T} \mathrm{~m}$.

on the outboard mid-plane, and has

$$
\cdots-I_{-1}=I_{0}=-I_{1}=I_{2}=-I_{3}=I_{4}=-I_{5} \cdots .
$$

Note that, since there is nothing special about the stability of the $2 / 1$ tearing mode, the results of our investigation are also relevant to tearing modes of other helicities. However, they cannot be applied to $m=1$ internal kink modes, which always require special treatment in tokamaks. ${ }^{17}$ Such modes are, therefore, specifically excluded from our investigation (i.e. $q_{0}>1$ in all of the examples considered) and will be dealt with in a separate publication.

\section{Effect of an RMP field}

The interaction of tearing modes with an external magnetic perturbation is governed by the $\mathrm{C}^{+}$Vector [see Eqs. (1)], which is written

$$
\mathrm{C}^{+}=\sum_{m} I_{m} \mathbf{C}^{m+}
$$

[see Eq. (A.44)]. If there are $N$ rational surfaces in the plasma then the $\mathrm{C}^{m+}$ are $1 \times N$ vectors with real components $C_{j}^{m+}$ (for $j=1$ to $N$ ). The component $C_{j}^{m+}$ drives tearing amplitude on surface $j$ [see Eqs. (12)], and also gives rise to a nonlinear electromagnetic torque acting at this surface [see Eq. (13)]. 
Consider the interaction of a static RMP field satisfying Eq. (52) with the 2/1 tearing mode. The locking torque exerted at the $q=2$ surface (labelled surface 1 in the following) is parameterized by $\left|I_{2} C_{1}^{2+}\right|^{2}$ [see Eq. (13)]. It is demonstrated in Sec. A.V that $C_{j}^{m+}$ is the expansion coefficient (in the vacuum region $r>a$ ) of the free boundary basis tearing mode associated with surface $j$ in the $m$ th well behaved (as $R \rightarrow \infty$ ) toroidal ring function. This result, which is obtained from a consideration of toroidal angular momentum conservation, is only valid in the absence of a conducting shell.

In the adopted ordering scheme $C_{1}^{2+}$, which describes the interaction of a predominately $m=2$ external perturbation with a tearing mode of the same dominant helicity, is expanded

$$
C_{1}^{2+}=\lambda^{(0)}+\lambda^{(1)} \epsilon^{2}+\lambda^{(2)} \epsilon^{2} \beta_{p}^{2}+\lambda^{(3)} \epsilon^{2} \beta_{p}+\lambda^{(4)} E_{a}^{2}+\lambda^{(3)} T_{a}^{2}+\mathcal{O}\left(\epsilon^{3}\right),
$$

where $\lambda^{(0)}$ is the cylindrical limit, $\lambda^{(1)}$ is a toroidicity correction, $\lambda^{(2)}$ is a pressure correction, $\lambda^{(3)}$ is a correction due to combined toroidal and pressure effects, $\lambda^{(4)}$ is an ellipticity correction, and $\lambda^{(5)}$ is a triangularity correction. Here, $E_{a} \equiv E(a)$ is the edge ellipticity parameter, and $T_{a} \equiv T(a)$ is the edge triangularity parameter [see Eqs. (47)]. In general, the free boundary basis tearing modes can only be calculated by solving the full coupled ideal MHD equations (A.6) in the outer region. This is achieved for the large aspect ratio, low- $\beta$, weakly shaped equilibria described in Sec. IV.B using the T7 code. ${ }^{13}$

Figure 3 shows the expansion coefficients of $C_{1}^{2+}$ evaluated from the T7 code as a function of $q_{a}$ in the range 2.5 to 5.2 , for $q_{0}=1.01$ and $k=4$. Figure 4 shows values of $C_{1}^{2+}$ calculated for an example equilibrium with $\epsilon=0.15, E_{a}=0.15, T_{a}=0.05$, and $\beta_{p}=0.0$ or 1.0.

It can be seen from Fig. 3 that the cylindrical part of $C_{1}^{2+}$ decays slowly as $q_{a}$ is increased. This is quite understandable, since as the edge- $q$ is increased (at constant central- $q$ ) the $q=2$ surface moves deeper inside the plasma, so the interiction with a fixed external perturbation is likely to get progressively weaker duc to range effects. The toroidal correction is found to increase the locking torque exerted at $q=2$ (i.e. increase $C_{1}^{2+}$ ) for $q_{a}<3$, but changes 
discontinuously as the $q=3$ surface enters the plasma so that the torque is decreased by toroidicity for $q_{a}>3$. The pressure and toroidal/pressure corrections strongly reinforce the locking torque for $q_{a}<3$, but become negligible for $q_{a}>3$. The ellipticity correction increases the locking torque for $q_{a}<4$, but changes discontinuously as the $q=4$ surface enters the plasma so that the torque is decreased by ellipticity for $q_{a}>4$. Finally, the triangularity correction increases the locking torque at small edge- $q$ (i.e. $q_{a} \lesssim 2.7$ ), decreases the torque at moderate and large edge- $q$, and behaves discontinuously as the $q=5$ surface enters the plasma.

The presence of an ideal mode rational surface (i.e. a surface on which there is no driven tearing or twisting amplitude, due to rotation effects) situated between the $q=2$ surface and the plasma boundary has the effect of 'shielding' the $2 / 1$ tearing mode from the applied RMP field to some extent, so that there is a discontinuity in $C_{1}^{2+}$ each time such a surface enters the plasma. It can be seen from Fig. 4 that at low- $\beta_{p}$ this shielding effect is rather weak, since the discontinuous changes of $C_{1}^{2+}$ at $q_{a}=3,4$, and 5 are nearly invisible. However, at high $-\beta_{p}$ the shielding of the $2 / 1$ tearing mode by an ideal $q=3$ surface becomes more appreciable, yielding a significant drop in $C_{1}^{2+}$ at $q_{a}=3$.

Note that a locked rational surface has no shielding effect whatsoever. This can be demonstrated by assuming that the $q=3$ surface is locked (at the tearing resonance) whenever it lies inside the plasma. According to the analysis of Sec. III.C.5, in this situation the locking torque exerted at $q=2$ is parameterized by $\left|I_{2} \tilde{C}_{1}^{2+}\right|^{2}$, where $\tilde{C}_{1}^{2+}=C_{1}^{2+}$ for $q_{\mathrm{a}}<3$ and

$$
\tilde{C}_{1}^{2+}=C_{1}^{2+}+\frac{E_{12}^{+}}{\left(-E_{22}^{+}\right)} C_{2}^{2+}
$$

for $q_{a}>3$. Figure 5 shows $\tilde{C}_{1}^{2+}$ evaluated as a function of $q_{a}$ for $q_{0}=1.01, k=4, \epsilon=0.15$, $E_{a}=0.15$, and $T_{a}=0.05$, with $\beta_{p}=0.0$ and 1.0. It can be seen by comparison with Fig. 4 that a locked $q=3$ surface does not shield the $2 / 1$ tearing mode from the applied RMP field, since there is no discontinuous reduction in $\tilde{C}_{1}^{2+}$ as the surface enters the plasma. 


\section{E. Effect of an error field}

Consider the interaction of an error field satisfying Eq. (53) with the $2 / 1$ tearing mode. The locking torque exerted at the $q=2$ surface is parameterized by $\left|I_{2} C_{1}^{\text {out }+}\right|^{2}$, where

$$
C_{1}^{\text {out }+}=\cdots-C_{1}^{-1+}+C_{1}^{0+}-C_{1}^{1+}+C_{1}^{2+}-C_{1}^{3+}+C_{1}^{4+}-C_{1}^{5+} \cdots
$$

Note that the superscript 'out' refers to the outboard location of the coils generating the

error field. In the adopted ordering scheme $C_{1}^{m \neq 2+}$, which governs the interaction of a tearing mode and an external perturbation with different dominant poloidal mode numbers, is expanded

$$
C_{1}^{m \neq 2+}=\lambda^{(1)} \epsilon+\lambda^{(2)} \epsilon \beta_{p}+\lambda^{(3)} E_{a}+\lambda^{(4)} T_{a}+\mathcal{O}\left(\epsilon^{2}\right)
$$

If the dominant poloidal mode numbers differ by unity, then only $\lambda^{(1)}$ and $\lambda^{(2)}$ are nonzero. If the mode numbers differ by two, or three, then only $\lambda^{(3)}$, or $\lambda^{(4)}$, are nonzero, respectively. If they differ by more than three then $C_{1}^{m \neq 2+}$ is negligible.

Figure 6 shows the expansion coefficients of the $C_{1}^{m \neq 2+}$ calculated from the $T 7$ code as functions of $q_{a}$ for $m$ in the range -1 to 5 , with $q_{0}=1.01$ and $k=4$. The expansion coefficients for $C_{1}^{0+}$ are found to be identically zero (and are, therefore, not plotted), indicating that an $m=0$ external perturbation does not interact with tearing modes. The quantity $C_{1}^{1+}$ is found to have no pressure correction. This is due to the peculiar nature of the $m=1$ harmonic in tokamak plasmas. The expansion coefficients of $C_{1}^{3+}$ have large discontinuities at $q_{a}=3$, showing that the presence of an ideal $q=3$ surface in the plasma shields the $q=2$ surface from the influence of a predominantly $m=3$ external perturbation. The expansion coefficients of $C_{1}^{4+}$ and $C_{1}^{5+}$ have similar large discontinuities at $q_{a}=4$ and 5 , respectively.

Figure 7 shows $C_{1}^{\text {outt }}$ as a function of $q_{a}$ for $q_{0}=1.01, k=4, \epsilon=0.15, E_{a}=0.15$, and $T_{a}=0.05$, with $\beta_{p}=0.0$ and 0.2 . Note the 'staircase' variation with edge- $q$, characterized by relatively little change in $C_{1}^{\text {out }}$ between integer $q_{a}$ values, with large discontinuities at $q_{a}=3$ and 4 . The discontinuous reduction in $C_{1}^{\text {outt }}$ at $q_{a}=3$ occurs because, as soon 
as it enters the plasma, an ideal $q=3$ surface shields the $q=2$ surface from the $m=3$ component of the error field. Likewise, the discontinuous reduction at $q_{a}=4$ occurs because an ideal $q=4$ surface shields out the $m=4$ component of the error field. Note that for a broad spectrum error field the discontinuities are $\mathcal{O}(\epsilon)$, whereas the narrow spectrum RMP field studied in Sec. IV.D only yields $\mathcal{O}\left(\epsilon^{2}\right)$ discontinuities. This accounts for the markedly different variations of $C_{1}^{2+}$ and $C_{1}^{\text {out }+}$ with edge- $q$ shown in Figs. 4 and 6 , respectively. Note that Fig. 7 would exhibit no discontinuities at $q_{a}=3$ and 4 were the $q=3$ and 4 surfaces locked, since locked surfaces possess no shielding properties (see Sec. IV.D).

In experiments, the locking torque exerted at $q=2$ by a fixed error field is conveniently parameterized by the line-averaged density below which a static $2 / 1$ island is induced in the plasma. The larger the density, the larger the torque, and vice versa. ${ }^{4}$ For narrow spectrum RMP fields the minimum density is observed to decrease smoothly with increasing edge-q, ${ }^{4}$ implying a smoothly decreasing locking torque similar to that shown in Fig. 4. On the other hand, for the broad spectrum JET error field (see Table II) the minimum density hardly changes between integer $q_{a}$ values, but decreases discontinuously as the $q=3$ and $q=4$ surfaces enter the plasma. ${ }^{10}$ This behaviour is consistent with the staircase variation of the locking torque shown in Fig. 7, assuming that both the $q=3$ and $q=4$ surfaces are unlocked. Note that discontinuous behaviour is only observed in limiter plasmas. For separatrix plasmas, which lie beyond the scope of this paper, there is no observed stepwise variation of minimum density as the ratio of plasma current to toroidal field is changed. ${ }^{10}$

The effect of ideal rational surfaces on the locking torque exerted at $q=2$ is largely dependent on the relative phases of the different poloidal harmonics of the error field. For an error field source located on the outboard mid-plane the phases are such that the torque increases as the $q=3$ and $q=4$ surfaces leave the plasma. Consider an error field produced by a source localized on the inboard mid-plane $(\theta=0)$. For such a field the locking torque 
exerted at $q=2$ is parameterized by $\left|I_{2} C_{1}^{\text {in }+}\right|^{2}$, where

$$
C_{1}^{\mathrm{in}+}=\cdots C_{1}^{-1+}+C_{1}^{0+}+C_{1}^{1+}+C_{1}^{2+}+C_{1}^{3+}+C_{1}^{4+}+C_{1}^{5+} \cdots
$$

Figure 8 shows $C_{1}^{\text {int }}$ as a function of $q_{a}$ for $q_{0}=1.01, k=4, \epsilon=0.15, E_{a}=0.15$, and $T_{a}=0.05$, with $\beta_{p}=0.0$ and 0.2 . It can be seen that in this case the phases are such that the locking torque decreases discontinuously as the $q=3$ surface leaves the plasma, while increasing at $q_{a}=4$ and 5. Thus, in this situation the behaviour at $q_{a}=3$ is opposite to that for the outboard error field source. Even more complicated behaviour is obtained if the error field source is located significantly above or below the mid-plane.

Figure 9 shows $C_{1}^{\text {out }+}$ as a function of $q_{a}$ for $q_{0}=1.1, k=4, \epsilon=0.15, E_{a}=0.15$, and $T_{a}=0.05$, with $\beta_{p}=0.0$ and 0.2 . The increased central $-q$ value brings the plasma very close to the $3 / 1$ ideal external kink stability boundary at $q_{a} \sim 3$. It can be seen that the torque exerted at $q=2$ increases very markedly as $q_{a} \rightarrow 3$ from below. This effect is due to the $m=3$ component of the error field, as is demonstrated by its sudden disappearance as soon as the $q=3$ surface, which effectively shields out the $m=3$ error field, enters the plasma. Figure 9 suggests that the locking torque exerted on the plasma by a fixed error field is likely to become very large close to an ideal external kink stability boundary. This effect may offer an explanation of recent DIII-D results which imply a substantial increase in the error field locking torque exerted at $q=2$ as the Troyon $\beta$-limit is approached. ${ }^{5}$

\section{Calculation of the $\mathrm{C}^{-}$Vector}

The interaction of twisting modes with an external perturbation is governed by the $\mathrm{C}^{-}$Vector [see Eqs. (1)], which is written

$$
\mathbf{C}^{-}=\sum_{m} I_{m} \mathbf{C}^{m-}
$$

[see Eq. (A.44)|. If there are $N$ rational surfaces in the plasma then the $\mathbf{C}^{m-}$ are $1 \times N$ vectors with real components $C_{j}^{m-}$ (for $j=1$ to $N$ ). The component $C_{j}^{m-}$ drives twisting 
amplitude on surface $j$ [see Eqs. (12)], and also gives rise to a nonlinear electromagnetic locking torque acting at this surface [see Eq. (13)].

It is demonstrated in Sec. A.V that $C_{j}^{m-}$ is the expansion coefficient (in the vacuum region $r>a$ ) of the free boundary basis twisting mode associated with surface $j$ in the $m$ th well behaved (as $R \rightarrow \infty$ ) toroidal ring function. As is described in Sec. A.IV of Ref. 12 the basis twisting modes can be built up out of solutions of the cylindrical tearing mode equation. Let $\psi_{m_{j} \pm 1}^{L}(r)$ be a solution of this equation (for poloidal mode number $m_{j} \pm 1$ ) in the interval $0 \leq r<r_{j}$ which satisfies the physical boundary conditions at $r=0$, is zero at the $m_{j} \pm 1 / n$ rational surface if it lies in the region $0<r<r_{j}$, and is unity just inside the $m_{j} / n$ rational surface at $r_{j-}$. Likewise, let $\psi_{m, \pm 1}^{R}(r)$ be a solution of the cylindrical tearing mode equation in the interval $r>r_{j}$ which satisfies free boundary conditions for $r>a$, is zero at the $m_{j} \pm 1 / n$ rational surface if it lies in the region $r_{j}<r \leq a$, and is unity just outside the $m_{j} / n$ rational surface at $r_{j+}$. It is useful to define the quantities

$$
\begin{aligned}
& \Delta_{m_{j \pm 1}}^{L}=\left[r \frac{d \psi_{m_{j} \pm 1}^{L}}{d r}\right]_{r_{j-}}, \\
& \Delta_{m, \pm 1}^{R}=\left[r \frac{d \psi_{m_{j \pm 1}}^{R}}{d r}\right]_{r_{j+}} .
\end{aligned}
$$

According to Ref. 12, the $j$ th basis twisting mode is built up out of $\mathcal{O}(\epsilon)$ of the $m_{j} \pm 1$ poloidal harmonics,

$$
\begin{aligned}
\psi_{m, \pm 1}(r)=-\frac{\pi}{2} \frac{\alpha_{j}}{s_{j}}\left(m_{j} \pm 1\right)[ & \frac{\Delta_{m_{j} \pm 1}^{R} \pm\left(m_{j} \pm 1\right)\left(1+s_{j}\right)}{\Delta_{m_{j} \pm 1}^{R}-\Delta_{m_{j} \pm 1}^{L}} \psi_{m_{j} \pm 1}^{L}(r) \\
& \left.+\frac{\Delta_{m_{j} \pm 1}^{L} \pm\left(m_{j} \pm 1\right)\left(1+s_{j}\right)}{\Delta_{m_{j} \pm 1}^{R}-\Delta_{m_{j} \pm 1}^{L}} \psi_{m_{j} \pm 1}^{R}(r)\right],
\end{aligned}
$$

with only $\mathcal{O}\left(\epsilon^{2}\right)$ of the other poloidal harmonics (including the central $m_{j}$ harmonic). Here, $\alpha_{j}$ is the $\mathcal{O}(\epsilon)$ pressure gradient parameter at rational surface $j$ [see Eq. (4)], and $s_{j} \equiv$ $\left(r q^{\prime} / q\right)_{r}$, is the local magnetic shear. It follows from Sec. A.V that

$$
C_{j}^{m_{j} \pm 1-}=C_{m, \pm 1} \alpha_{j}
$$


where

$$
c_{m, \pm 1}=-\frac{\pi}{s_{j}} \frac{\Delta_{m, \pm 1}^{L} \pm\left(m_{j} \pm 1\right)\left(1+s_{j}\right)}{\Delta_{m, \pm 1}^{R}-\Delta_{m, \pm 1}^{L}} \psi_{m, \pm 1}^{R}(a),
$$

since $\psi_{m_{j} \pm 1}^{L}(a)=0$ by definition [see Eqs. (A.27), (A.32), (A.46), and (A.48)]. Note that the $C_{j}^{m_{k}-}$ are $\mathcal{O}\left(\epsilon^{2}\right)$ for $m_{k} \neq m_{j} \pm 1$. Thus, the $j$ th basis twisting mode (resonant with poloidal mode number $m_{j}$ ) is most strongly affected by external perturbations whose dominant poloidal mode numbers are $m_{j} \pm 1$.

Consider the interaction of a static RMP field satisfying Eq. (52) with the $2 / 1$ twisting mode. The locking torque exerted at the $q=2$ surface is parameterized by $\left|I_{2} C_{1}^{2-}\right|^{2}$ [see Eq. (13)]. It is clear from the above that $C_{1}^{2-} \sim \mathcal{O}\left(\epsilon^{2}\right)$, so this torque is $\mathcal{O}\left(\epsilon^{4}\right)$. The locking torque exerted at $q=2$ due to interaction with the $2 / 1$ tearing mode is parameterized by $\left|I_{2} C_{1}^{2+}\right|^{2}$ and is $\mathcal{O}(1)$ (see Sec. IV.D). We conclude that in this case the locking torque due to interaction with the $2 / 1$ twisting mode is negligible compared to that associated with the $2 / 1$ tearing mode.

Consider the interaction of an error field satisfying Eq. (53) with the $2 / 1$ twisting mode. The locking torque exerted at the $q=2$ surface is parameterized by $\left|I_{2} C_{1}^{\text {out }-}\right|^{2}$, where

$$
C_{1}^{\text {out }-}=-C_{1}^{1-}-C_{1}^{3-}+\mathcal{O}\left(\epsilon^{2}\right)
$$

The quantities $C_{1}^{1-}$ and $C_{1}^{3-}$ can be evaluated using a cylindrical tearing mode code via Eqs. (63) and (64). It is found that $C_{1}^{1-}$ is zero (i.e. the $2 / 1$ twisting mode is not affected by a predominantly $1 / 1$ external perturbation). This result is due to the peculiar nature of the $m=1$ harmonic in tokamak plasmas and is not general. For instance, the $3 / 1$ twisting mode is affected by a predominantly $2 / 1$ external perturbation. It is also found that $C_{1}^{3-}$ drops discontinuously to zero as the $q=3$ surface enters the plasma, because an ideal $q=3$ surface completely shields the $2 / 1$ twisting mode from the influence of a predominantly $3 / 1$ external perturbation. So, in the adopted ordering scheme

$$
C_{1}^{\text {out }-}=\lambda \epsilon \beta_{\mathrm{p}}
$$




\begin{tabular}{|l|l|}
\hline$q_{a}$ & $\lambda$ \\
\hline 2.5 & 6.73 \\
2.6 & 7.14 \\
2.7 & 7.46 \\
2.8 & 7.75 \\
2.9 & 8.27 \\
2.99 & 9.61 \\
\hline
\end{tabular}

Tabla III: Values of $\lambda$ [defined in Eq. (66)] as a function of $q_{a}$, for $q_{0}=1.01$.

where $\lambda$ is zero for $q_{a}>3$. Values of $\lambda$ for $q_{a}$ in the range 2.5 to 3.0 , and $q_{0}=1.01$, are given in Table III. It is clear that the locking torque due to interaction with the $2 / 1$ twisting mode is zero for $q_{a}>3$, and is $\mathcal{O}\left(\epsilon \beta_{p}\right)^{2}$ for $q_{a}<3$. This should be compared with the locking torque due to interaction with the $2 / 1$ tearing mode which is $\mathcal{O}(1)$, with $\mathcal{O}(\epsilon)$ discontinuities at integer edge- $q$ (see Sec. IV.E). Thus, if $\epsilon \beta_{p} \ll 1$ the locking torque associated with the externally driven $2 / 1$ tearing mode again dominates that due to the twisting mode.

\section{Summary}

We have examined the influence of a general static external magnetic perturbation on the stability of resistive modes in a tokamak plasma. There are three main parts to this investigation.

Firstly, the vacuum external perturbation must be expanded as a series of well behaved $($ as $r \rightarrow 0$ ) toroidal ring functions (see Sec. IV.C). A perturbation with a given toroidal mode number is fully specified by the complex coefficients of this expansion, which are denoted by the $I_{m}$, where $m$ is the number of poloidal nodes. Typical coefficients for a deliberately applied RMP field and an accidentally occurring error field are given in Tables I and II, respectively. The RMP field considered is designed to have a narrow spectrum with one particular resonant $I_{m}$ dominant, whereas error fields tend to have broad spectra.

Secondly, the dispersion relation for resistive modes in the presence of a general external 
perturbation must be derived (see appendix). The unperturbed dispersion relation takes the form of two coupled homogeneous $N \times N$ matrix equations, where $N$ is the number of rational surfaces in the plasma (resonant with a given toroidal mode number). In the presence of an external perturbation these equations acquire right-hand sides, denoted by the $1 \times N$ vectors $\mathrm{C}^{ \pm}$. The $\mathrm{C}^{+}$Vector characterizes the response of tearing parity modes to the external perturbation, whereas the $\mathrm{C}^{-}$Vector characterizes the response of twisting parity modes. The $\mathrm{C}^{ \pm}$Vectors are decomposed $\mathrm{C}^{ \pm}=\sum_{m} I_{m} \mathrm{C}^{m \pm}$. It is demonstrated in the appendix that the components of the $\mathbf{C}^{m \pm}$ vectors are obtainable from the asymptotic behaviour (as $R \rightarrow \infty$ ) of the free boundary basis tearing and twisting modes (in the absence of the external perturbation). This result follows from a consideration of toroidal angular momentum conservation, but is only valid in the absence of a conducting shell.

Lastly, the resistive dispersion relation must be solved to give the tearing and twisting amplitudes driven in the plasma by the external perturbation (see Sec. III). The electromagnetic locking torque exerted at rational surface $j$ is proportional to $\left|C_{j}^{+}\right|^{2}$ and $\left|C_{j}^{-}\right|^{2}$, where $C_{j}^{ \pm}$are the $j$ th components of the $C^{ \pm}$Vectors. Considering the simplest case where there is only a single rational surface in the plasma and $\left|C_{j}^{+}\right|^{2} \gg\left|C_{j}^{-}\right|^{2}$, we find that as soon as $\left|C_{j}^{+}\right|$ exceeds the critical value needed to half the natural frequency for tearing modes at surface $j$, this frequency suddenly drops to a value very close to zero, and there is a dramatic increase in the driven tearing amplitude. This process is termed 'locking.' Prior to locking there is very little driven tearing amplitude at surface $j$.

The problem becomes more complicated if $\left|C_{j}^{-}\right|^{2} \gtrsim\left|C_{j}^{+}\right|^{2}$, so that the torque due to driven twisting amplitude at surface $j$ is comparable to that due to driven tearing amplitude, but this is unlikely to occur in low- $\beta$ plasmas (see Sec. V). When there is more than one rational surface in the plasma, locking torques are exerted simultaneously at all surfaces, and a change in rotation induced at a given surface can influence the other surfaces via the action of bulk plasma viscosity. We have derived a set of coupled nonlinear equations which describe the 
response of each rational surface to a general external perturbation (see Sec. III.D). These equations have been solved for an example case with two rational surfaces in the plasma (see Sec. III.C). In general, we find that the critical electromagnetic torque required to lock surface $j$ is rediced if torques are exerted at any other surfaces. Furthermore, locking occurs when the natural frequency of tearing modes at surface $j$ has been reduced to a critical value which is now somewhat less than half of its unperturbed value.

The T7 toroidal tearing mode $\operatorname{code}^{13}$ has been extended to evaluate the $\mathrm{C}^{\mathrm{m}+}$ vectors (see Sec. IV). This allows us to construct the $\mathrm{C}^{+}$Vector, given the complex amplitudes, $I_{m}$, which characterize the external perturbation. We have considered two idealized external perturbations. The first has $I_{m}=I$ with $I_{m^{\prime} \neq m}=0$ (where $I$ is a constant) and represents a typical narrow spectrum RMP field produced by external saddle coils. The second has $I_{m}=(-1)^{m} I$ and represents an error field produced by a localized source on the outboard mid-plane.

Consider the locking torque exerted at a typical low mode number rational surface such as $q=2$. We find that ideal rational surfaces located between this surface and the plasma edge tend to 'shield' it from the locally resonant component of the applied external perturbation. For instance, an ideal $3 / 1$ surface shields out the $m=3$ component. This effect leads to a di,continuous variation of the locking torque with edge- $q$. There is a sudden change in the torque as the $q=3$ surface enters the plasma and shields the $m=3$ component of the applied perturbation. There is a similar sudden change as the $q=4$ surface enters the plasma. For a narrow spectrum RMP field the discontinuous changes in the locking torque at rational edge- $q$ are $\mathcal{O}\left(\epsilon^{2}\right)$ (where $\epsilon$ is the inverse aspect ratio) and are not a dominant feature of the variation with edge-q. However, for a broad spectrum error field the discontinuous changes are $\mathcal{O}(\epsilon)$ and tend to be the dominant feature of the edge- $q$ variation. For an error field source located on the outboard mid-plane there is a 'stajrcase' variation, with relatively little change between integer edge- $q$ values, but strong reductions at $q=3$ and 4 (as $q_{a}$ 
is increased). Such behaviour has been observed experimentally. ${ }^{10}$ An error field source located on the inboard mid-plane, or significantly off the mid-plane, generally gives rise to more complicated variation of the locking torque with edge- $q$ (see Fig. 8). We find that locked rational surfaces have no shielding effect, so that there is no sudden change in the torque as a locked surface is brought into the plasma. We also find that the locking torque exerted by a fixed error field becomes very large close to an ideal external kink stability boundary. We speculate that this effect may account for the observed significant reduction in the error field strength needed to induce locking at $q=2$ close to the Troyon $\beta$-limit in DIII-D. ${ }^{5}$

The components of the $\mathrm{C}^{m-}$ vector can be evaluated using solutions of the cylindrical tearing mode equaticn (see Sec. V). We find that in low- $\beta$ plasmas, where $\epsilon \beta_{p} \ll 1$, the locking torques due to externally driven twisting modes are generally negligible compared to the torques associated with driven tearing modes. However, this is unlikely to remain the case in high- $\beta$ plasmas, where $\epsilon \beta_{p} \gtrsim \mathcal{O}(1)$.

\section{Acknowledgments}

The authors gratefully acknowledge the assistance of Dr. G.M. Fishpool in obtaining data on JET field errors. This work was jointly funded by the UK Department of Trade and Industry, Euratom, and the U.S. Department of Energy contract \# DE-FG05-80ET-53088. 


\section{A Physics of the Outer Region}

\section{A. The marginally stable ideal MHD equations}

The coordinate system $(r, \theta, \phi)$, where $\phi$ is the toroidal angle, $\theta$ is an angle-like variable in the poloidal plane, and $r$ is a flux surface label with dimensions of length, is chosen so that the magnetic field lines appear straight. The Jacobian for these coordinates is given by ${ }^{22}$

$$
j=(\nabla r \wedge \nabla \theta \cdot \nabla \phi)^{-1}=\frac{r R^{2}}{R_{0}},
$$

where $R$ is the major radius, and $R_{0}$ is the average major radius of the outermost plasma flux surface. For an axisymmetric equilibrium the magnetic field $B$ can be written

$$
\mathrm{B}=B_{0} R_{0}[f(r) \nabla \phi \wedge \nabla r+g(r) \nabla \phi]
$$

where $B_{0}$ is the vacuum magnetic field strength at $R=R_{0}$. The safety factor, the slope of the field lines in the $\theta-\phi$ plane, is then given by

$$
q(r)=\frac{r g(r)}{R_{0} f(r)} .
$$

The perturbed magnetic field $\delta \mathbf{B}$ is completely specified by two sets of flux surface functions, $\psi_{m}(r)$ and $Z_{m}(r)$, where

$$
\begin{aligned}
\delta \mathrm{B} \cdot \nabla r & =\mathrm{i} \frac{R_{0}^{2}}{R^{2}} \sum_{m} \frac{\psi_{m}(r)}{r} \exp [\mathrm{i}(m \theta-n \phi)], \\
R_{0} \delta \mathrm{B} \cdot \nabla \phi & =n \frac{R_{0}^{2}}{R^{2}} \sum_{m} \frac{\left[Z_{m}(r)+\lambda_{m} \psi_{m}(r)\right]}{R_{0}(m-n q)} \exp [\mathrm{i}(m \theta-n \phi)],
\end{aligned}
$$

with

$$
\lambda_{m}=\left[m \frac{\mu_{0} r p^{\prime}}{B_{0}^{2} f^{2}}\left\langle\frac{R^{2}}{R_{0}^{2}} \frac{1}{|\nabla r|^{2}}\right\rangle-\frac{m}{n}(m-n q) \frac{R_{0} g^{\prime}}{f}\left\langle\frac{1}{|\nabla r|^{2}}\right\rangle\right] / L_{m}^{m}
$$




$$
\begin{aligned}
L_{m}^{m} & =\left[m^{2}\left\langle\frac{1}{|\nabla r|^{2}}\right\rangle+n^{2} \frac{r^{2}}{R_{0}^{2}}\right], \\
\langle\cdots\rangle & \equiv \frac{1}{2 \pi} \oint(\cdots) d \theta .
\end{aligned}
$$

Here, ' denotes differentiation with respect to $r$, and $p(r)$ is the plasma pressure.

Throughout the bulk of the plasma the perturbed field is governed by the marginally stable equations of ideal magnetohydrodynamics (MHD), which take the form:

$$
\begin{array}{r}
r \frac{d \psi_{m}}{d r}=\frac{L_{m}^{m} Z_{m}}{(m-n q)}+\sum_{k \neq 0} \frac{\left(L_{m}^{m+k} Z_{m+k}+M_{m}^{m+k} \psi_{m+k}\right)}{(m+k-n q)}, \\
(m-n q) r \frac{d}{d r}\left[\frac{Z_{m}}{(m-n q)}\right]=\frac{P_{m}^{m} \psi_{m}}{(m-n q)}+\sum_{k \neq 0} \frac{\left(N_{m}^{m+k} Z_{m+k}+P_{m}^{m+k} \psi_{m+k}\right)}{(m+k-n q)} .
\end{array}
$$

The coefficients $L_{m}^{m+k}, M_{m}^{m+k}, N_{m}^{m+k}$ and $P_{m}^{m+k}$ are evaluated for a general low- $\beta$, large aspect ratio tokamak equilibrium in Ref. 13. The ordering adopted is such that the Shafranov shift and departure from circularity of plasma flux surfaces are both $\mathcal{O}(\epsilon)$ with respect to the average minor radius of the outermost plasma flux surface, $a$, where $\epsilon=a / R_{0} \ll 1$ is the inverse aspect ratio. This implies that $L_{m}^{m} \sim \mathcal{O}(1)+\mathcal{O}\left(\epsilon^{2}\right)$ and $L_{m}^{m+k} \sim \mathcal{O}(\epsilon)$, with a similar ordering for the other coefficients. Coupling of harmonics of the perturbed field whose poloidal mode numbers differ by unity is effected by the Shafranov shift of flux surfaces, which is driven by toroidicity and the plasma pressure. Coupling of harmonics whose mode numbers differ by two or three is effected by flux surface ellipticity or triangularity, respectively. The ordering adopted for the Shafranov shift and flux surface shaping implies that $g=1+\mathcal{O}\left(\epsilon^{2}\right)$ and $\mu_{0} p / B_{0}^{2} \sim \mathcal{O}\left(\epsilon^{2}\right)$.

\section{B. The outer solution in the vicinity of a rational surface}

The marginally stable ideal MHD equations (A.6) become singular on flux surfaces where the safety facto: $q$ takes the rational value $m / r_{b}$. Such surfaces are termed rational surfaces resonant with poloidal mode number $m$. The most general expression for the resonant 
harmonic of the perturbed poloidal flux in the vicinity of a rational surface, radius $r_{m}$, is

$$
\begin{aligned}
\psi_{m} \simeq\left[A^{+}+\left(\frac{\nu \pi}{2}\right)\right. & \left.A^{-} \operatorname{sgn}(x)\right]|x|^{\nu}[1+\cdots \mid \\
& +\frac{1}{2}\left[B^{+}+\left(\frac{\nu \pi}{2}\right)^{-1} B^{-} \operatorname{sgn}(x)\right]|x|^{1-\nu}+C x+\cdots,
\end{aligned}
$$

where $x=r-r_{m}$, and

$$
\nu \simeq\left[-\frac{2 \mu_{0} r p^{\prime}}{B_{0}^{2} s^{2}}\left(1-q^{2}\right)\right]_{r_{m}}
$$

represents the effect of average field line curvature. ${ }^{23}$ Here, $s=\left(r q^{\prime} / q\right)_{r_{m}}$ is the local magnetic shear, and $A^{ \pm}, B^{ \pm}$, and $C$ are arbitrary constants.

The two ratios

$$
\Delta^{ \pm}(\omega)=\frac{B^{ \pm}}{A^{ \pm}}
$$

are completely determined by the solution of the even and odd parity Fourier transformed layer equations in the inner region, and are in general functions of the mode rotation frequency $\omega$ [where all layer quantities are assumed to vary like $\exp (-i \omega t)$ ].

\section{Basis tearing and twisting modes}

Suppose there are $N$ rational surfaces in the plasma (radii $r_{1}<r_{2} \cdots<r_{N}$ ), resonant with poloidal mode numbers $m_{1}, m_{2} \cdots m_{N}$ (for a fixed toroidal mode number $n$ ). It is useful to define the quantities ${ }^{12}$

$$
\begin{array}{r}
\Psi_{j}^{ \pm}=\left[\frac{\left(1-2 \nu_{j}\right)}{L_{m_{j}}^{m_{j}}}\right]_{r_{j}}^{1 / 2}\left(A^{ \pm}\right)_{j}, \\
\Delta \Psi_{j}^{ \pm}=\left[\frac{\left(1-2 \nu_{j}\right)}{L_{m_{j}}^{m_{j}}}\right]_{r_{j}}^{1 / 2}\left(B^{ \pm}\right)_{j},
\end{array}
$$

where $\nu_{j}$ is the Mercier index for surface $j$ [see Eq. (A.8)], $\Psi_{j}^{+}$is termed the 'tearing amplitude' at surface $j$, and $\Psi_{j}^{-}$is the associated 'twisting amplitude.' The tearing amplitude is 
sometimes referred to as the 'reconnected flux.' It follows from (A.9) that

$$
\Delta_{j}^{ \pm}(\omega)=\frac{\Delta \Psi_{j}^{ \pm}}{\Psi_{j}^{ \pm}},
$$

where $\Delta_{j}^{+}(\omega)$ is the tearing parity layer dispersion relation at surface $j$, and $\Delta_{j}^{-}(\omega)$ is the associated twisting parity dispersion relation. The toroidal electromagnetic torque acting in the vicinity of surface $j$ takes the form ${ }^{12}$

$$
\delta T_{\phi}\left(r_{j}\right)=\frac{2 n \pi^{2} R_{0}}{\mu_{0}} \times\left[\operatorname{Im}\left(\Delta_{j}^{+}\right)\left|\Psi_{j}^{+}\right|^{2}+\operatorname{Im}\left(\Delta_{j}^{-}\right)\left|\Psi_{j}^{-}\right|^{2}\right] .
$$

The system has $2 N$ degrees of freedom (i.e. two degrees for each rational surface in the plasma), so a general mode can be built up from a linear superposition of $2 N$ independent basis modes. It is convenient to define $N$ basis tearing modes, denoted $\psi_{j}^{+}$(for $j=1$ to $N$ ). These are solutions of Eqs. (A.6) which satisfy the physical boundary conditions at $r$ and $r=a$ and are subject to the additional constraints: ${ }^{12}$

$$
\begin{gathered}
\Psi_{k}^{+}=\delta_{k j}, \\
\Psi_{k}^{-}=0, \\
\Delta \Psi_{k}^{+}=E_{k j}^{+}, \\
\Delta \Psi_{k}^{-}=H_{j k} .
\end{gathered}
$$

Thus, the $j$ th basis tearing mode has unit tearing amplitude and zero twisting amplitude at surface $j$, with zero tearing or twisting amplitude at any other surface. It is also convenient to define $N$ basis twisting modes, denoted $\boldsymbol{\psi}_{j}^{-}$. These are solutions of Eqs. (A.6) which satisfy the physical boundary conditions and are subject to the constraints: ${ }^{12}$

$$
\begin{aligned}
& \Psi_{k}^{+}=0, \\
& \Psi_{k}^{-}=\delta_{k j},
\end{aligned}
$$




$$
\begin{aligned}
& \Delta \Psi_{k}^{+}=H_{k j}, \\
& \Delta \Psi_{k}^{-}=E_{k j}^{-} .
\end{aligned}
$$

Thus, the $j$ th basis twisting mode has unit twisting amplitude and zero tearing amplitude at surface $j$, with zero tearing or twisting amplitude at any other surface. Note that the quantities $E_{k j}^{ \pm}$and $H_{k j}$ must be real because the ideal MHD equations (A.6) contain no complex coefficients.

A general mode is written

$$
\psi=\sum_{k=1}^{N}\left(\Psi_{k}^{+} \psi_{k}^{+}+\Psi_{k}^{-} \psi_{k}^{-}\right)
$$

yielding the resistive mode dispersion relation ${ }^{26}$

$$
\begin{aligned}
&\left\{\Delta^{+}(\omega)-\mathbf{E}^{+}\right\} \Psi^{+}-\mathbf{H} \Psi^{-}=0=0 \\
&\left\{\boldsymbol{\Delta}^{-}(\omega)-\mathbf{E}^{-}\right\} \boldsymbol{\Psi}^{-}-\mathbf{H}^{\dagger} \boldsymbol{\Psi}^{+}=0 .
\end{aligned}
$$

In the above, $\mathbf{E}^{ \pm}$is the $N \times N$ real symmetric matrix of the $E_{i j}^{ \pm}$values, $\mathbf{H}$ is the $N \times N$ real matrix of the $H_{\mathrm{t} j}$ values and $\mathbf{H}^{\dagger}$ is its transpose, $\Delta^{ \pm}(\omega)$ is the $N \times N$ complex diagonal matrix of the $\Delta_{j}^{ \pm}(\omega)$ values, and $\Psi^{ \pm}$is the $1 \times N$ complex vector of the $\Psi_{j}^{ \pm}$values. It follows from Eqs. (A.12) and (A.16) that

$$
T_{\phi}=\sum_{j=1}^{N} \delta T_{\phi}\left(r_{j}\right)=0
$$

so there is zero net toroidal electromagnetic torque acting on an isolated plasma.

\section{The vacuum region}

In the vacuum region external to the plasma the perturbed magnetic field is written

$$
\delta \mathrm{B}=\mathrm{i} \nabla V,
$$


where the scalar magnetic potential $V$ can be expanded

$$
V(r, \theta, \phi)=\sum_{m} V_{m}(r) \exp [\mathrm{i}(m \theta-n \phi)] .
$$

It follows from Eqs. (A.4) that for $r>a$

$$
Z_{m}(r)=(m-n q) V_{m}(r)
$$

and

$$
\begin{aligned}
\psi_{m}(r)= & \sum_{k}\left\langle\frac{R^{2}}{R_{0}^{2}}|\nabla r|^{2} \exp (\mathrm{i} k \theta)\right\rangle r \frac{d V_{m+k}}{d r}(r) \\
& -\sum_{k}\left\langle\frac{R^{2}}{R_{0}^{2}} \mathrm{i} r \nabla r \cdot \nabla \theta \exp (\mathrm{i} k \theta)\right\rangle(m+k) V_{m+k}(r) .
\end{aligned}
$$

In vacuum the scalar magnetic potential satisfies Laplace's equation

$$
\nabla^{2} V=0
$$

It is easily demonstrated that if $V^{a}$ and $V^{b}$ are two general solutions of Eq. (A.22) with the same toroidal mode number then

$$
\frac{d}{d r} \sum_{k}\left[\psi_{k}^{a}(r) V_{k}^{b}(r)-\psi_{k}^{b}(r) V_{k}^{a}(r)\right]=0,
$$

where the functions $\psi_{k}^{a}(r)$ are related to the functions $V_{k}^{a}(r)$ via Eq. (A.21).

It is convenient to define the general solution vector $\psi$, and the related vector $\mathrm{V}$, where the components $\psi_{k}(r)$ of $\psi$ are the harmonics of the perturbed poloidal flux, so that $\psi(r$, $\theta, \phi)=\sum_{k} \psi_{k}(r) \exp [\mathrm{i}(k \theta-n \phi)]$. Likewise, the components $V_{k}(r)$ of $\mathbf{V}$ are the harmonics of the scalar potential, so that $V(r, \theta, \phi)=\sum_{k} V_{k}(r) \exp [\mathrm{i}(k \theta-n \phi)]$. The components of $\psi$ and $\mathbf{V}$ are interrelated via Eq. (A.21). Let

$$
\left[\psi^{a}, \psi^{b}\right](r) \equiv \sum_{k}\left[\psi_{k}^{a}(r) V_{k}^{b}(r)-\psi_{k}^{b}(r) V_{k}^{a}(r)\right]
$$


where $\psi^{a}$ and $\psi^{b}$ are two general solution vectors. It follows from Eq. (A.23) that $\left[\boldsymbol{\psi}^{a}, \boldsymbol{\psi}^{b}\right](r)$ is independent of $r$ in the vacuum region.

In the absence of plasma the vacuum region extends to the magnetic axis $(r=0)$. In the region close to the axis

$$
\psi_{m} \simeq r \frac{d V_{m}}{d r}
$$

and Laplace's equation reduces to

$$
\frac{1}{r} \frac{d}{d r}\left(r \frac{d V_{m}}{d r}\right)-\frac{m^{2}}{r^{2}} V_{m} \simeq 0
$$

with solutions $V_{m}(r) \propto r^{ \pm|m|}$ for $m \neq 0$ and $V_{0}(r) \propto \ln r, r^{0}$. Let the vacuum basis solution $\mathbf{P}^{m}$ have components which satisfy (A.25) and (A.26), and reduce to

$$
\psi_{m}(r)=\left(\frac{r}{a}\right)^{-|m|}, \quad \psi_{l \neq m}(r)=0
$$

in the limit $r \rightarrow 0$. For the special case $m=0$, the components of the basis solution $\mathbf{P}^{0}$ reduce to

$$
\psi_{0}(r)=1, \quad \psi_{l \neq 0}(r)=0
$$

at the magnetic axis. Likewise, let the vacuum basis solution $\mathbf{Q}^{\mathbf{m}}$ have components which satisfy (A.25) and (A.26), and reduce to

$$
\psi_{m}(r)=\left(\frac{r}{a}\right)^{+|m|}, \quad \psi_{l \neq m}(r)=0
$$

in the limit $r \rightarrow 0$. For the special case $m=0$, the harmonics of the scalar potential derived from the elements of $\mathbf{Q}^{0}$ [via Eq. (A.25)] reduce to

$$
V_{0}(r)=1, \quad V_{l \neq 0}(r)=0
$$

at the magnetic axis. Note that only the $\mathbf{Q}^{m}$ basis solutions are well behaved in the limit $r \rightarrow 0$. 
It follows from Eqs. (A.23), (A.24), and (A.27) to (A.30), that

$$
\begin{aligned}
& {\left[\mathbf{P}^{m}, \mathbf{P}^{l}\right](r)=0,} \\
& {\left[\mathbf{Q}^{m}, \mathbf{Q}^{l}\right](r)=0,} \\
& {\left[\mathbf{P}^{m}, \mathbf{Q}^{l}\right](r)=\delta^{m l} h_{m}}
\end{aligned}
$$

throughout the vacuum region, where

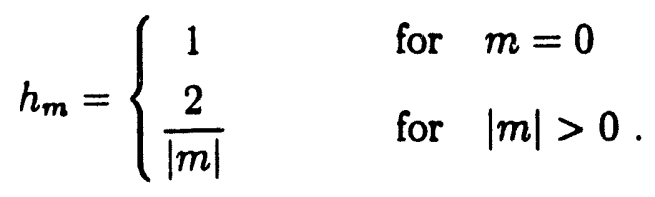

The scalar magnetic potential associated with the vacuum basis solution $\mathbf{P}^{m}$ takes the form $^{13}$

$$
\begin{aligned}
V(\mu, \eta, \phi)=\frac{h_{m} \Gamma\left(\frac{|m|}{2}-\frac{n}{2}+\frac{1}{4}\right) \Gamma\left(\frac{|m|}{2}-\frac{n}{2}+\frac{3}{4}\right)}{(-1) 2^{n+1}(|m|-1) !(-\epsilon)^{-|m|}} & \\
& \times \sqrt{\cosh \mu-\cos \eta} P_{m-\frac{1}{2}}^{n}(\cosh \mu) \exp [i(m \eta-n \phi)],
\end{aligned}
$$

and the potential associated with the basis solution $\mathbf{Q}^{\mathbf{m}}$ is written

$$
\begin{aligned}
& V(\mu, \eta, \phi)=\frac{2^{|m|+\frac{1}{2}}(|m|-1) !}{(-1)^{n} \sqrt{\pi \Gamma}\left(|m|+n+\frac{1}{2}\right)(-\epsilon)^{|m|}} \\
& \quad \times \sqrt{\cosh \mu-\cos \eta} Q_{m-\frac{1}{2}}^{n}(\cosh \mu) \exp [i(m \eta-n \phi)] .
\end{aligned}
$$

Here, $(\mu, \eta, \phi)$ are standard toroidal coordinates,

$$
\begin{aligned}
& R=R_{0} \frac{\sinh \mu}{\cosh \mu-\cos \eta}, \\
& Z=R_{0} \frac{\sin \eta}{\cosh \mu-\cos \eta},
\end{aligned}
$$


while $P_{a}^{b}$ and $Q_{a}^{b}$ are associated Legendre functions, and $k !=1$ for $k \leq 0$. Note that only the $\mathbf{P}^{m}$ basis solutions are well behaved as $R \rightarrow \infty$.

The most general solution of the ideal MHD equations in the vacuum region $(r>a)$ is written

$$
\psi=\sum_{m}\left(a_{m} \mathbf{P}^{m}+b_{m} \mathbf{Q}^{m}\right)
$$

where $a_{m}$ and $b_{m}$ are arbitrary complex constants. A particular vacuum solution $\psi$ can be resolved into components of the basis solutions $\mathbf{P}^{m}$ and $\mathbf{Q}^{m}$ via [see Eqs. (A.31)]

$$
\begin{aligned}
& a_{m}=h_{m}^{-1}\left[\psi, \mathbf{Q}^{m}\right](r>a), \\
& b_{m}=-h_{m}^{-1}\left[\boldsymbol{\psi}, \mathbf{P}^{m}\right](r>a) .
\end{aligned}
$$

Finally, it can be demonstrated that the total toroidal electromagnetic torque acting on the plasma satisfies ${ }^{13}$

$$
T_{\phi}(r>a) \equiv \int_{0}^{a} \oint \oint R^{2} \nabla \phi \cdot \delta \mathbf{J} \wedge \delta \mathbf{B} j d r d \theta d \phi=\frac{2 n \pi^{2} R_{0}}{\mu_{0}} \times \sum_{m} h_{m} \operatorname{Im}\left(a_{m}^{*} b_{m}\right),
$$

where $\delta \mathbf{J}$ is the perturbed current.

\section{E. The effect of an external helical magnetic field}

Consider the effect of a nonrotating externally imposed helical magnetic field on the resistive dispersion relation for a free boundary plasma.

The most general solution in the absence of plasma is written

$$
\psi^{\text {vac }}\left(r<r_{\text {coil }}\right)=\sum_{m} I_{m} \mathbf{Q}^{m}(r)
$$

where the $I_{m}$ are arbitrary complex constants, and $r_{\text {coil }}$ is the innermost radius of the external conductors used to generate the applied field. The scalar magnetic potential of the imposed 
field in the absence of plasma, $V^{\text {ext }}$, is easily calculated from the currents flowing in these conductors. The corresponding values of the $I_{m}$ are given by [see Eq. (A.34)]

$$
\begin{aligned}
I_{m}=\frac{(-1)^{n} \sqrt{\pi} \Gamma\left(|m|+n+\frac{1}{2}\right)(-\epsilon)^{|m|}}{2^{|m|+\frac{1}{2}}(|m|-1) ! Q_{m-\frac{1}{2}}^{n}(\cosh \mu)} \\
\quad \times \oint \oint \frac{V^{\text {ext }}(\mu, \eta, \phi) \exp [-i(m \eta-n \phi)]}{\sqrt{\cosh \mu-\cos \eta}} \frac{d \eta}{2 \pi} \frac{d \phi}{2 \pi},
\end{aligned}
$$

where the integration is carried out on a toroidal surface, $\mu=$ constant [see Eqs. (A.35)], lying inside the external conductors.

The most general solution in the presence of plasma is written

$$
\psi\left(r<r_{\text {coil }}\right)=\sum_{j=1}^{N}\left[\Psi_{j}^{+} \psi_{j}^{+}(r)+\Psi_{j}^{-} \psi_{j}^{-}(r)\right]+\sum_{m} I_{m} \chi^{m}(r)
$$

The solution $\chi^{m}$ has the following properties:

$$
\begin{gathered}
\Psi_{k}^{ \pm}=0 \\
\Delta \Psi_{k}^{ \pm}=C_{k}^{m \pm}
\end{gathered}
$$

for $k=1$ to $N$, and

$$
\chi^{m}\left(r_{\text {coil }}>r>a\right)=\sum_{k} h_{k}^{-1} \alpha_{k}^{m} \mathbf{P}^{k}(r)+\mathbf{Q}^{m}(r) .
$$

Here, the $C_{k}^{m \pm}$ and $\alpha_{k}^{m}$ are arbitrary real constants. Thus, $\chi^{m}$ specifies the ideal response of the plasma to the external field associated with the vacuum basis solution $\mathbf{Q}^{\mathbf{m}}$.

It follows from (A.13), (A.14), (A.41), and (A.42) that the general externally driven resistive dispersion relation takes the form

$$
\begin{aligned}
\left\{\Delta^{+}(\omega)-\mathbf{E}^{+}\right\} \Psi^{+}-\mathbf{H} \boldsymbol{\Psi}^{-}=\mathbf{C}^{+} \equiv \sum_{m} I_{m} \mathbf{C}^{m+} \\
\left\{\boldsymbol{\Delta}^{-}(\omega)-\mathbf{E}^{-}\right\} \boldsymbol{\Psi}^{-}-\mathbf{H}^{\dagger} \boldsymbol{\Psi}^{+}=\mathbf{C}^{-} \equiv \sum_{m} I_{m} \mathbf{C}^{m-}
\end{aligned}
$$


where the $\mathrm{C}^{m \pm}$ are the $1 \times N$ real vectors of the $C_{k}^{m \pm}$ values.

Using Eqs. (A.12) and (A.44), the total toroidal electromagnetic torque acting on the plasma is given by

$$
\begin{aligned}
T_{\phi}(r>a)=\sum_{j=1}^{N} \delta T_{\phi}\left(r_{j}\right)= & \frac{2 n \pi^{2} R_{0}}{\mu_{0}} \times \\
& \sum_{m} \sum_{j=1}^{N} \operatorname{Im}\left[I_{m}\left\{C_{j}^{m+}\left(\Psi_{j}^{+}\right)^{*}+C_{j}^{m-}\left(\Psi_{j}^{-}\right)^{*}\right\}\right] .
\end{aligned}
$$

Since the basis tearing and twisting solutions, $\psi_{j}^{+}$and $\boldsymbol{\psi}_{j}^{-}$, are well behaved for $r>a$, their most general expansion in the vacuum region is [see Eq. (A.36)]

$$
\psi_{j}^{ \pm}(r>a)=\sum_{m} a_{m, j}^{ \pm} \mathbf{P}^{m}(r)
$$

where the $a_{m, j}^{ \pm}$are real constants. Equations (A.38), (A.41), (A.43), and (A.46) yield a second expression for the total toroidal electromagnetic torque acting on the plasma:

$$
\begin{aligned}
T_{\phi}(r>a)= & \frac{2 n \pi^{2} R_{0}}{\mu_{0}} \times \\
& \sum_{m} \operatorname{Im}\left[\sum_{j=1}^{N} h_{m} I_{m}\left\{a_{m, j}^{+}\left(\Psi_{j}^{+}\right)^{*}+a_{m, j}^{-}\left(\Psi_{j}^{-}\right)^{*}\right\}+\sum_{l} \alpha_{m}^{l} I_{m} I_{i}^{*}\right] .
\end{aligned}
$$

The identity of Eqs. (A.45) and (A.47) for arbitrary $\Psi_{j}^{ \pm}$and $I_{m}$ yields $\alpha_{m}^{b}=\alpha_{l}^{m}$ and

$$
\begin{aligned}
& C_{j}^{m+}=h_{m} a_{m, j}^{+} \equiv\left[\psi_{j}^{+}, \mathbf{Q}^{m}\right], \\
& C_{j}^{m-}=h_{m} a_{m, j}^{-} \equiv\left[\psi_{j}^{-}, \mathbf{Q}^{m}\right] .
\end{aligned}
$$

\section{F. Summary}

A general external helical magnetic perturbation can be resolved into components of the well behaved (as $r \rightarrow 0$ ) vacuum basis solutions $\mathbf{Q}^{m}$ [see Eq. (A.39)], so that it is completely specified by a set of complex amplitudes $I_{m}$. The $I_{m}$ are calculated from the scalar magnetic potential of the vacuum external perturbation using Eq. (A.40). 
The response of the plasma to an idealized perturbation made up of unit amount of the vacuum basis solution $\mathbf{Q}^{m}$ is determined by the vectors $\mathbf{C}^{m \pm}$ [see Eqs. (A.44)]. The components of these vertors can be obtained by resolving the free boundary basis tearing and twisting modes in the vacuum region $(r>a)$ into components of the well behaved (as $R \rightarrow \infty$ ) basis vacuum solutions $\mathbf{P}^{m}$, according to Eqs. (A.48). Thus, the response of the plasma to a general perturbation is determined by the vectors $\mathrm{C}^{ \pm}$[see Eq. (A.44)], whose components are obtained from the coefficients of the expansion of the external perturbation in the $\mathbf{Q}^{\mathbf{m}}$ (with no plasma), and the coefficients of the expansion of the basis plasma modes in the $\mathbf{P}^{m}$ (for $r>a$, with no external perturbation). 


\section{References}

1. J.T. Scoville, R.J. La Haye, A.G. Kellman, T.H. Osborne, R.D. Stambaugh, E.J. Strait, and T.S. Taylor, Nucl. Fusion 31, 875 (1991).

2. R. Fitzpatrick, and T.C. Hender, Phys. Fluids B 3, 644 (1991).

3. A.W. Morris, P.G. Carolan, R. Fitzpatrick, T.C. Hender, and T.N. Todd, Phys. Fluids B 4, 413 (1992).

4. T.C. Hender, R. Fitzpatrick, A.W. Morris, P.G. Carolan, R.D. Durst, T. Edlington, J. Ferreira, S.J. Fielding, P.S. Haynes, J. Hugill, I.J. Jenkins, R.J. La Haye, B.J. Parham, D.C. Robinson, T.N. Todd, M. Valovič, and G. Vayakis, Nucl. Fusion 32, 2091 (1992).

5. R.J. La Haye, A.W. Hyatt, and J.T. Scoville, Nucl. Fusion 2, 2119 (1992).

6. R. Fitzpatrick, in Theory of Fusion Plasmas, Proceedings of the Joint Varenna-Lausanne International Workshop, Varenna 1992, (Società Italiana di Fisica, Bologna, 1992), p. 147 .

7. R.J. La Haye, R. Fitzpatrick, T.C. Hender, A.W. Morris, J.T. Scoville, and T.N. Todd, Phys. Fluids B 4, 2098 (1992).

8. T.H. Jensen, A.W. Leonard, and A.W. Hyatt, Phys. Fluids B 5, 1239 (1993).

9. R. Fitzpatrick, Nucl. Fusion 33, 1049 (1993).

10. G.M. Fishpool, and P.S. Haynes, Field Error Instabilities in JET, to appear in Nuclear Fusion (1994). 
11. M. Huguet, in Steady-State Operation of Fusion Plasmas, Proceedings of the 11th European Tokamak Workshop, Noordwijk (FOM-Nieuwegein, 1993), p. 547.

12. R. Fitzpatrick, Stability of Coupled Tearing and Twisting Modes in Tokamaks, IFS Report \#644, Submitted to Physics of Plasmas.

13. R. Fitzpatrick, R.J. Hastie, T.J. Martin, and C.M. Roach, Nucl. Fusion 33, 1533 (1993).

14. H.P. Furth, J. Killeen, and M.N. Rosenbluth, Phys. Fluids 6, 459 (1963).

15. W.A. Newcomb, Ann. Phys. 10, 232 (1960).

16. T.C. Hender, and S.C. Cowley, Phys. Fluids B 1, 2194 (1989).

17. M.N. Bussac, D. Edery, R. Pellat, and J.L. Soulé, in Plasma Physics and Controlled Nuclear Fusion Research 1976, Proceedings of the 6th International Conference, Berchtesgaden (IAEA, Vienna, 1977), Vol. 1, p. 607.

18. S.C. Cowley, and R.J. Hastie, Phys. Fluids 31, 426 (1988).

19. R. Fitzpatrick. in Theory of Fusion Plasmas, Proceedings of the Joint Varenna-Lausanne International Workshop, Varenna 1992, (Società Italiana di Fisica, Bologna, 1992), p. 147.

20. P.H. Rutherford, Phys. Fluids 16, 1903 (1973).

21. A.W. Morris, P.G. Carolan, R. Fitzpatrick, T.C. Hender, R. O'Connell, T.N. Todd, and M. Valovič, in Controlled Fusion and Plasma Physics, Proceedings of the 20th European Conference, Lisbon 1993 (European Physical Society, Geneva, 1993), Vol 1, p. 227.

22. M.N. Bussac, R. Pellat, D. Edery, and J.L. Soulé, Phys. Rev. Lett. 35, 1638 (1975). 
23. C. Mercier, Nucl. Fusion 1, 47 (1960).

24. A.H. Glasser, J.M. Greene, and J.L. Johnson, Phys. Fluids 18, 875 (1975). 


\section{Figure Captions}

1. Bifurcation curves for locking of an external perturbation to the tearing and twisting resonances at a general rational surface. $y^{+}$[defined in Eq. (19d)] is the normalized locking torque due to the tearing resonance, and $y^{-}$[also defined in Eq. (19d)] is the normalized locking torque due to the twisting resonance. The solid curves correspond to locking to the tearing resonance, whereas the dashed curves correspond to locking to the twisting resonance. Curves are shown for various different values of the unperturbed normalized frequency $f_{1}$ [defined in Eq. (19b)].

2. (a)-(e) Locking thresholds (for the tearing resonances) in a plasma containing two rational surfaces. The variables $\alpha^{2}$ and $\beta^{2}$ are proportional to the locking torques exerted at surfaces 1 and 2, respectively. These torques are normalized with respect to the threshold torques for each surface taken in isolation. Curves are plotted for various values of the parameter $\lambda$, which depends on the relative positions of the two rational surfaces. The parameter $\kappa$ measures the ratio of the unperturbed natural frequencies at the two surfaces. Figure 2(a) shows data for $\kappa=1 / 4$; Fig. 2(b) shows data for $\kappa=1 / 2$; Fig. 2(c) shows data for $\kappa=1$; Fig. 2(d) shows data for $\kappa=2$; Fig. 2(e) shows data for $\kappa=4$.

3. The expansion coefficients of $C_{1}^{2+}$ evaluated as a function of edge- $q$, for $q_{0}=1.01$ and $k=4$. The various graphs show the cylindrical limit $\left[\lambda^{(0)}\right.$ in Eq. (55)], the toroidal correction $\left[\lambda^{(1)}\right.$ in Eq. (55)], the pressure correction $\left[\lambda^{(2)}\right.$ in Eq. (55)], the toroidal/pressure correction $\left[\lambda^{(3)}\right.$ in Eq. (55)], the ellipticity correction $\left[\lambda^{(4)}\right.$ in Eq. (55)], and the triangularity correction $\left[\lambda^{(5)}\right.$ in Eq. (55)].

4. The parameter $C_{1}^{2+}$ evaluated as a function of edge- $q$, for $q_{0}=1.01$ and $k=4$, with $\epsilon=0.15, E_{a}=0.15$, and $T_{a}=0.05$. Data is shown for $\beta_{p}=0.0(\square)$, and $\beta_{p}=1.0(\bigcirc)$. 
5. The parameter $\tilde{C}_{1}^{2+}$ evaluated as a function of edge- $q$, for $q_{0}=1.01$ and $k=4$, with $\epsilon=0.15, E_{a}=0.15$, and $T_{a}=0.05$. Data is shown for $\beta_{p}=0.0(\square)$, and $\beta_{p}=1.0(O)$.

6. Expansion coefficients of the $C_{1}^{m \neq 2+}$ evaluated as a function of edge- $q$, for $q_{0}=1.01$ and $k=4$. The various graphs show the toroidal correction $\left[\lambda^{(1)}\right.$ in Eq. (58)], the pressure correction $\left[\lambda^{(2)}\right.$ in Eq. (58)], the ellipticity correction $\left[\lambda^{(3)}\right.$ in Eq. (58)], and the triangularity correction $\left[\lambda^{(4)}\right.$ in Eq. (58)]. Data is shown for $C_{1}^{-1+}(\diamond), C_{1}^{1+}(\Delta)$, $C_{1}^{3+}(\nabla), C_{1}^{4+}(\square)$, and $C_{1}^{3+}(O)$.

7. The parameter $C_{1}^{\text {out }+}$ evaluated as a function of edge- $q$, for $q_{0}=1.01$ and $k=4$, with $\epsilon=0.15, E_{a}=0.15$, and $T_{a}=0.05$. Data is shown for $\beta_{p}=0.0(\square)$, and $\beta_{p}=0.2(O)$.

8. The parameter $C_{1}^{\text {int }}$ evaluated as a function of edge- $q$, for $q_{0}=1.01$ and $k=4$, with $\epsilon=0.15, E_{a}=0.15$, and $T_{a}=0.05$. Data is shown for $\beta_{p}=0.0(\square)$, and $\beta_{p}=0.2(O)$.

9. The parameter $C_{1}^{\text {out }}$ evaluated as a function of edge- $q$, for $q_{0}=1.1$ and $k=4$, with $\epsilon=0.15, E_{a}=0.15$, and $T_{a}=0.05$. Data is shown for $\beta_{p}=0.0(\square)$, and $\beta_{p}=0.2(O)$. 
$I_{ \pm}$

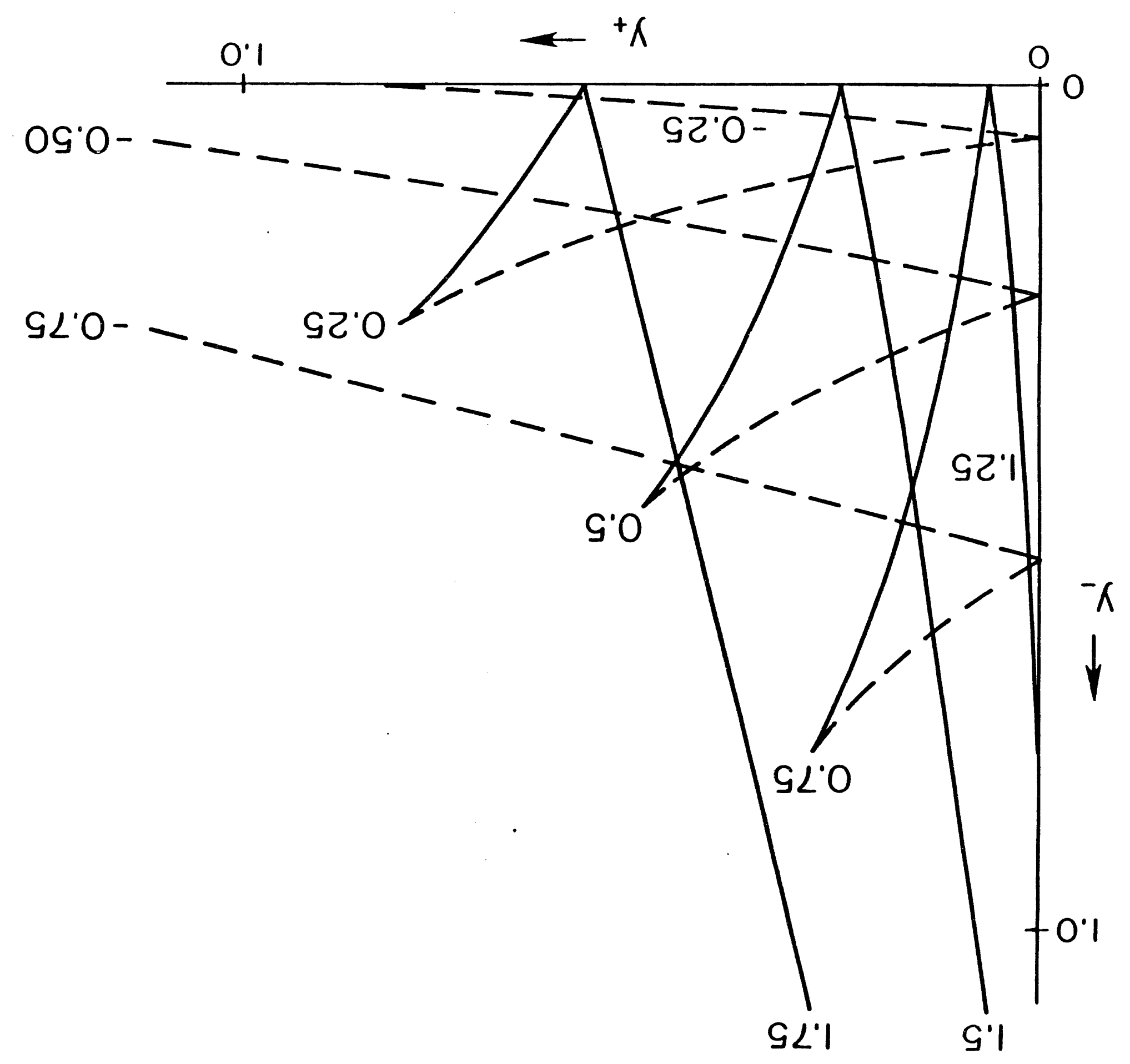




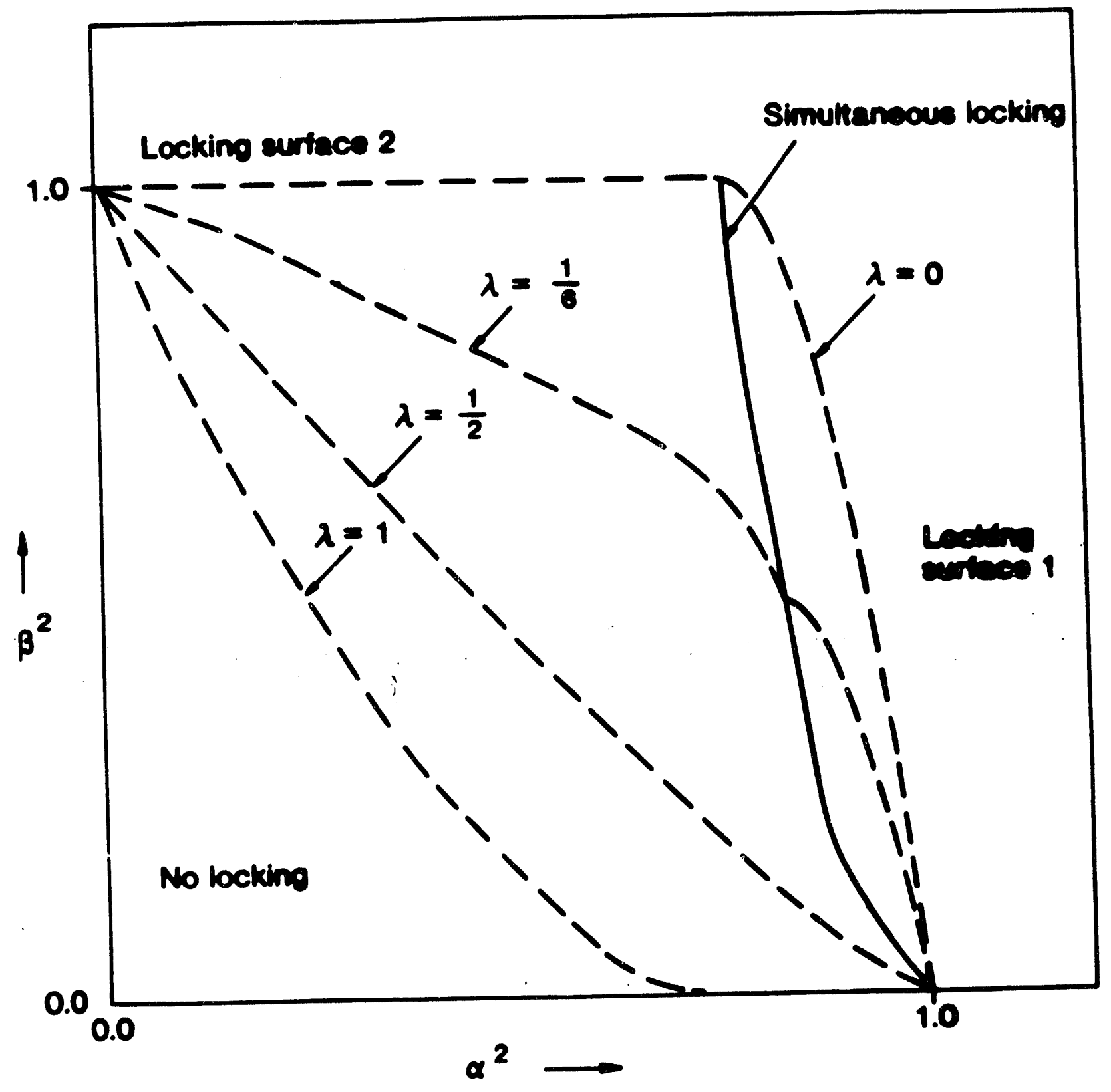

Fio. $2(a)$ 


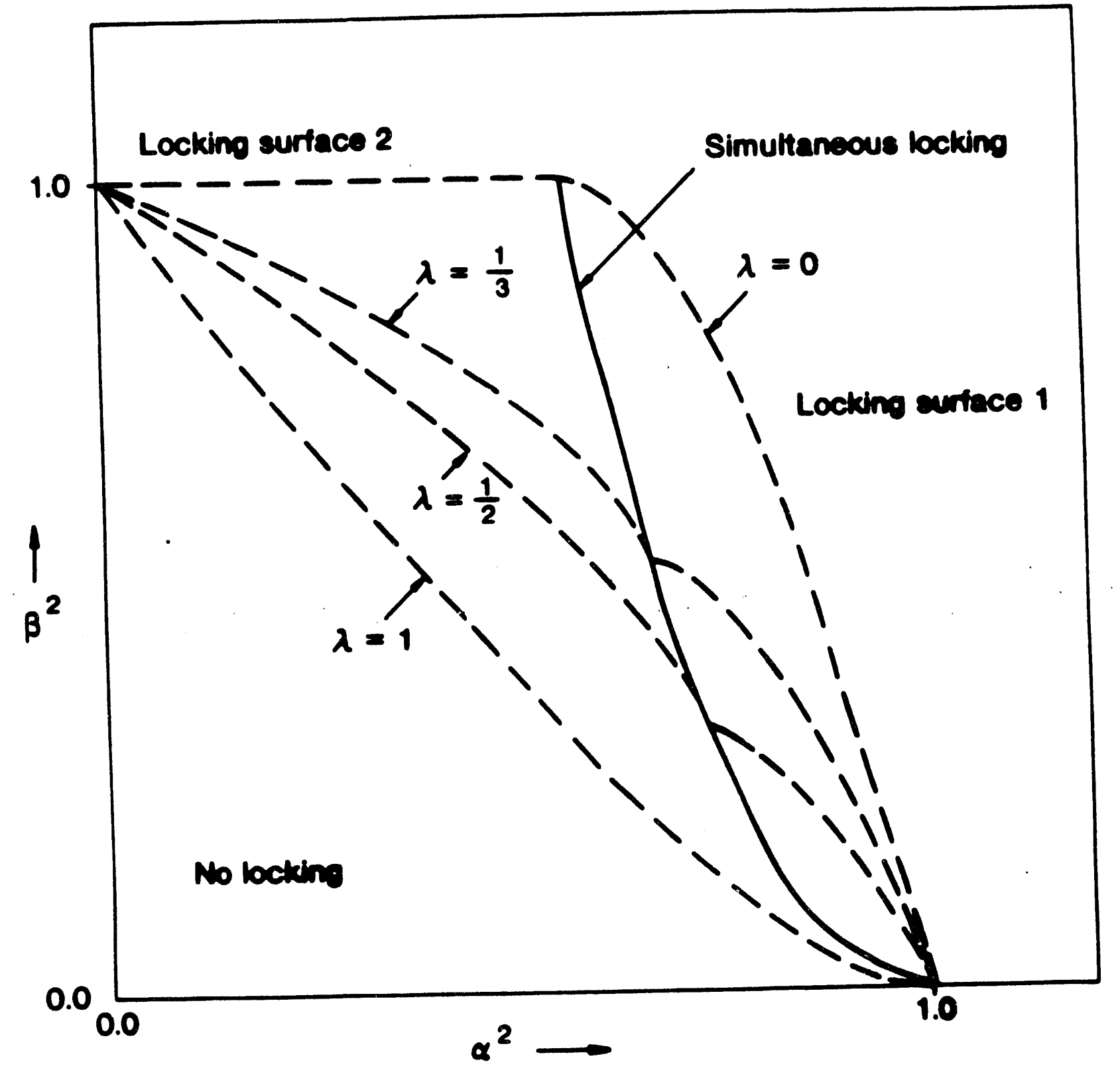

Fig. $2(b)$ 


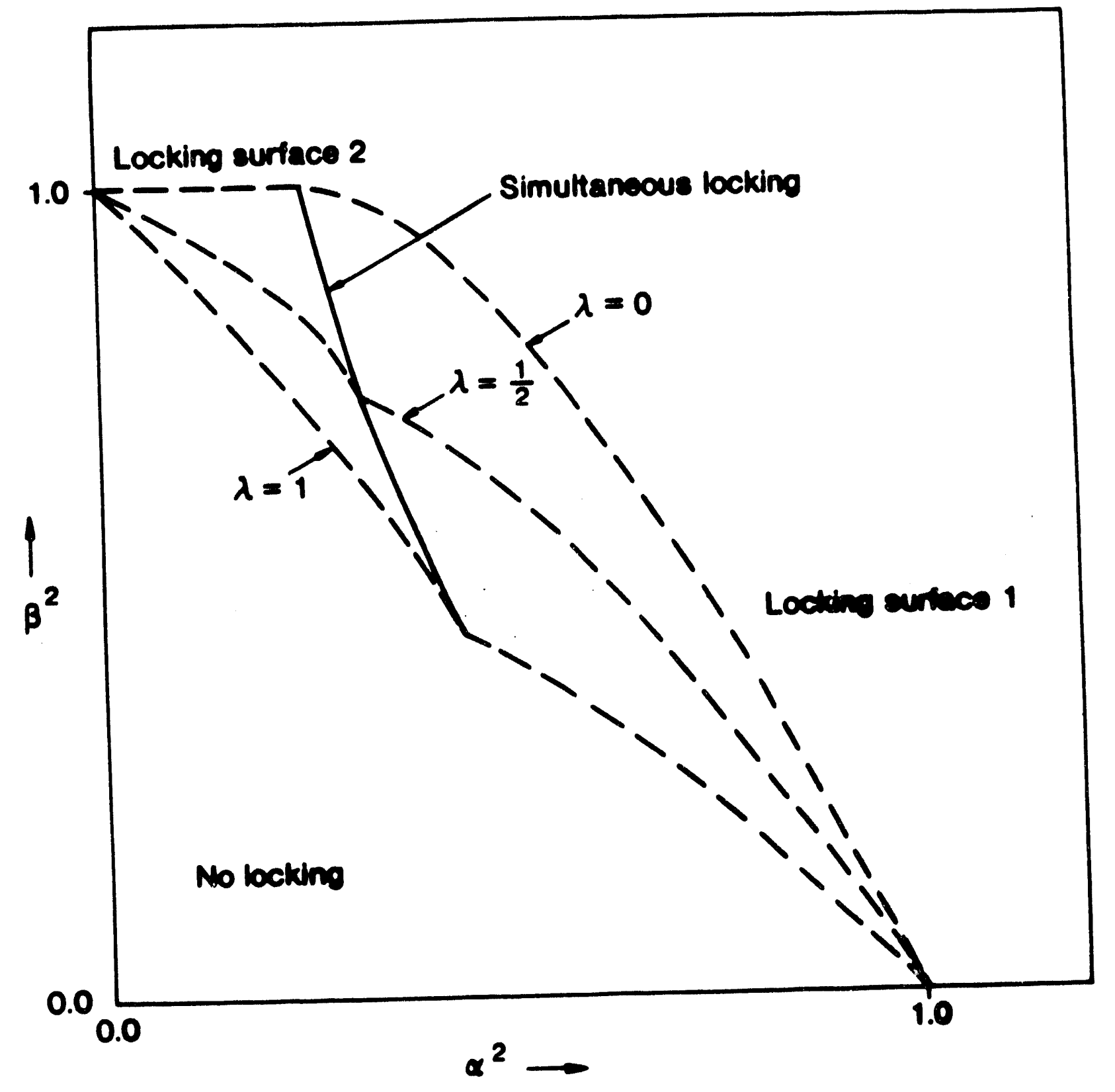

Fig. $2(c)$ 


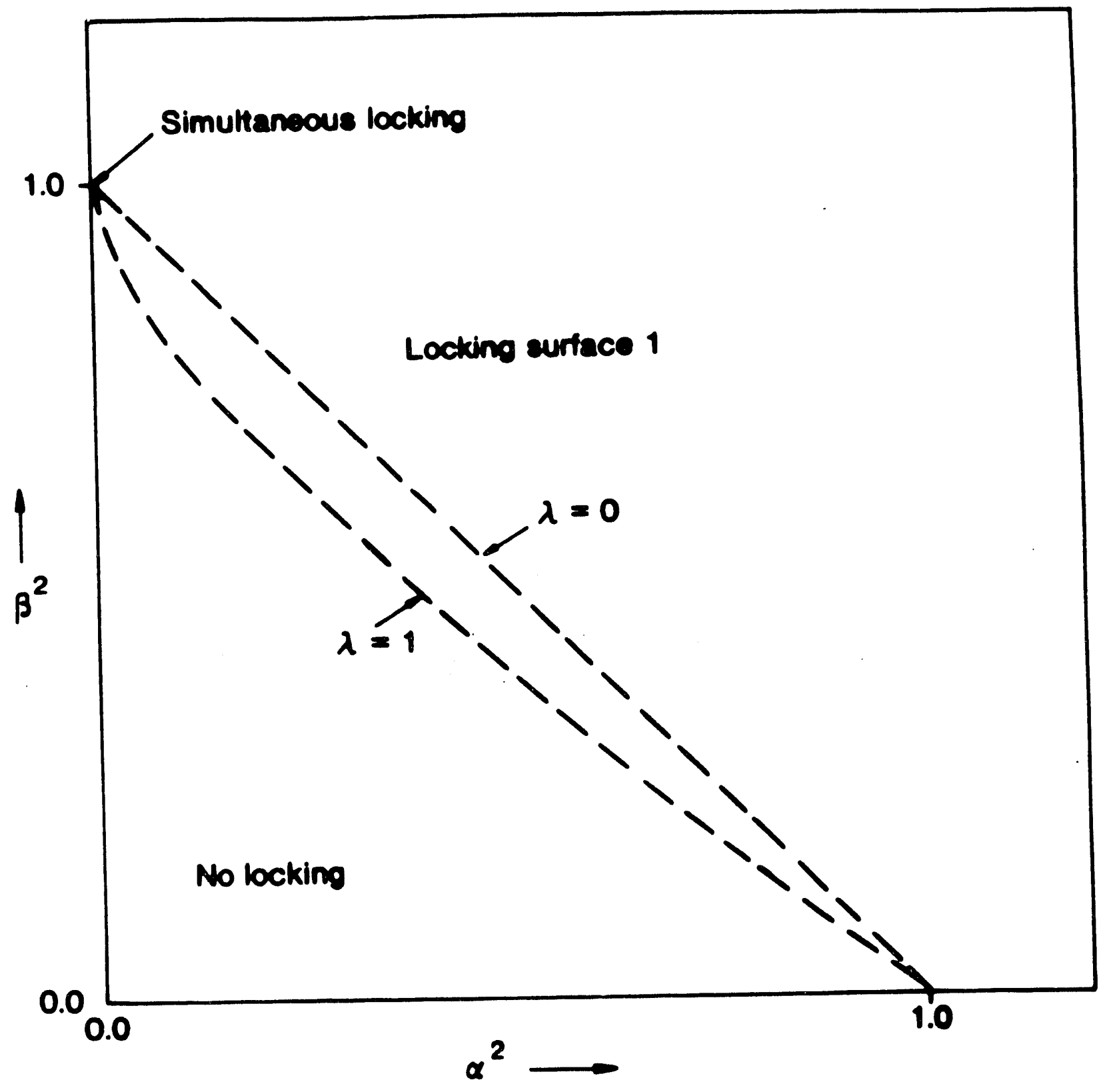

Fig.2(d) 


\section{(o) 0}

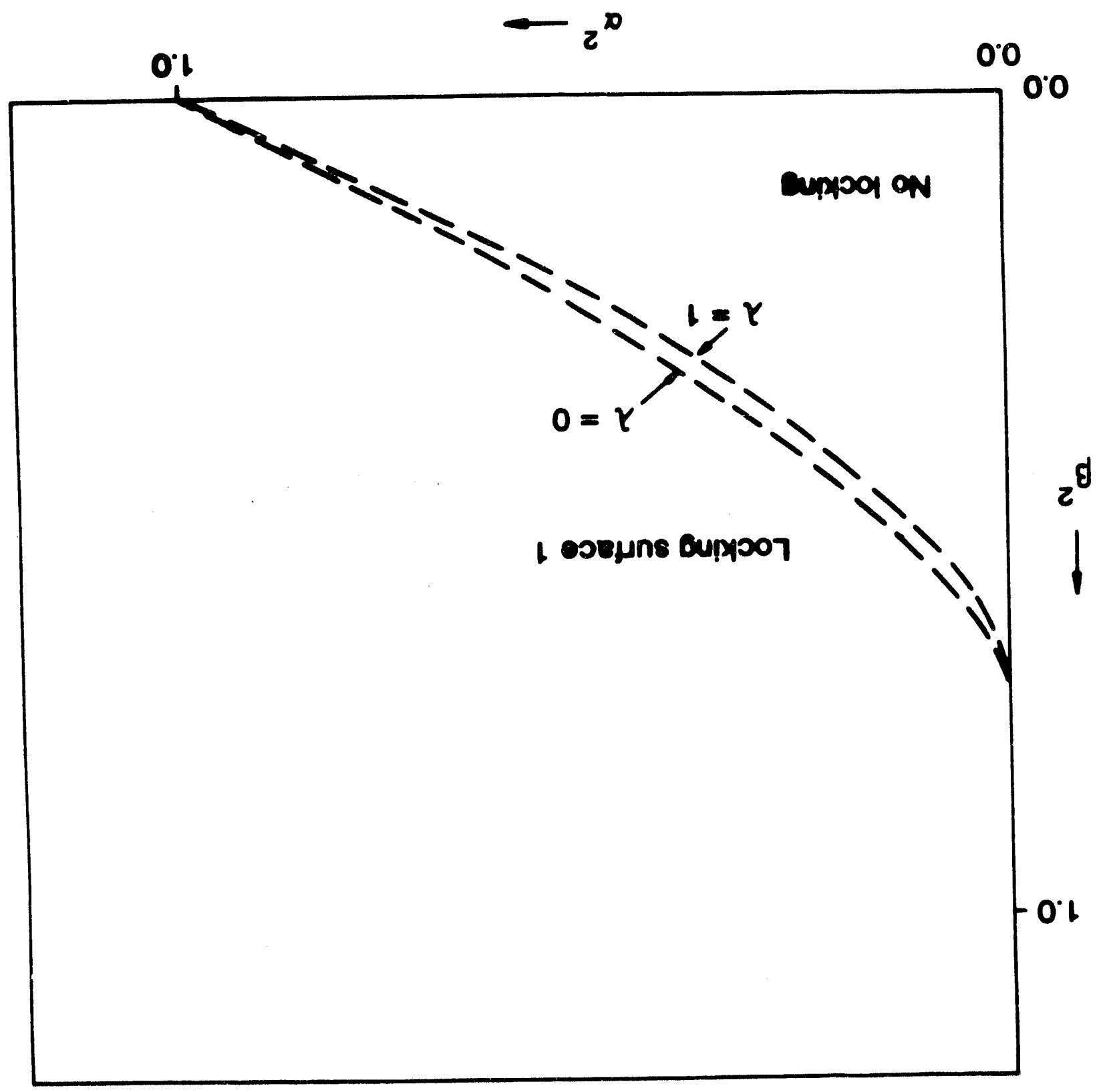



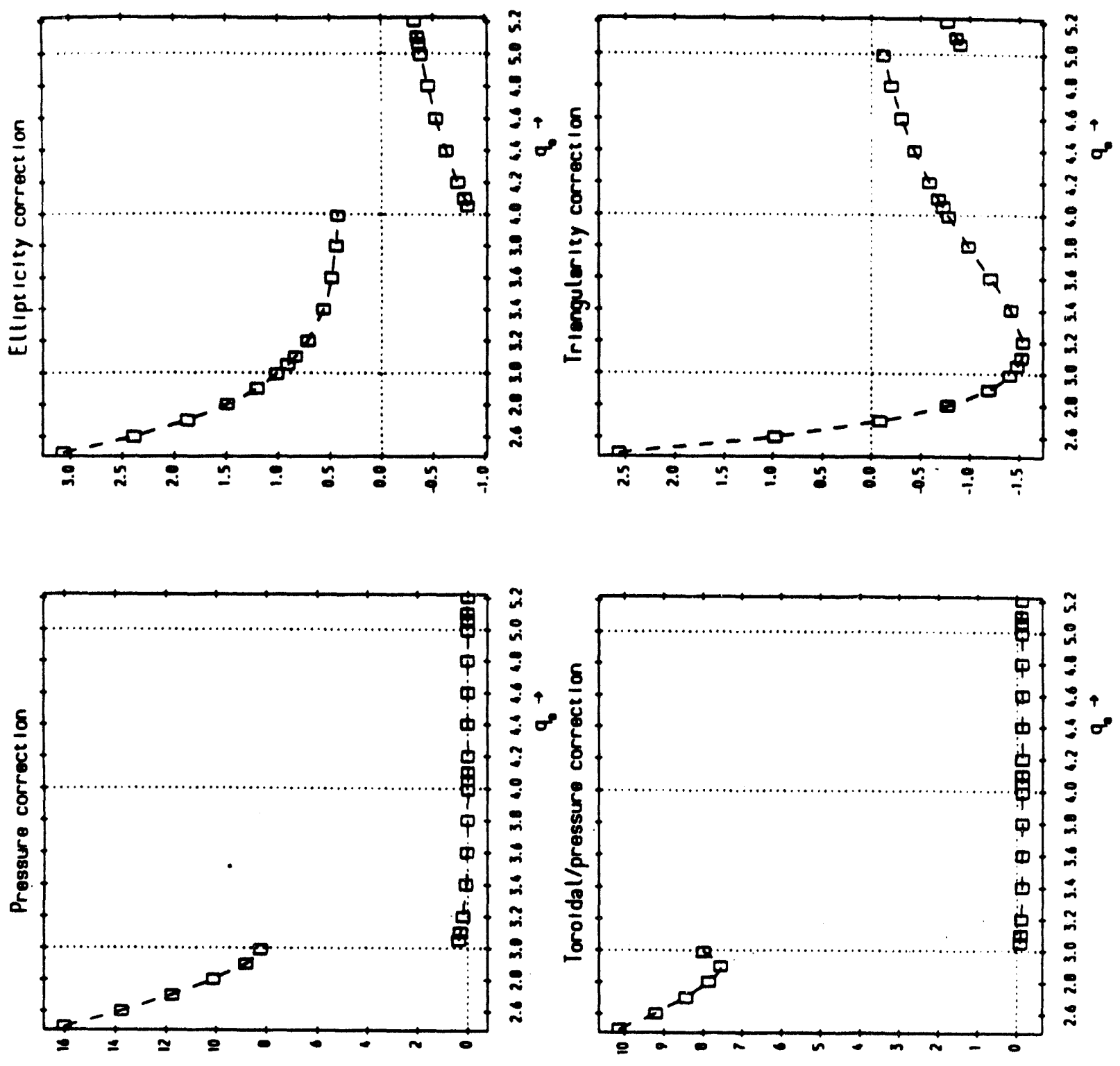

$N$
$W$

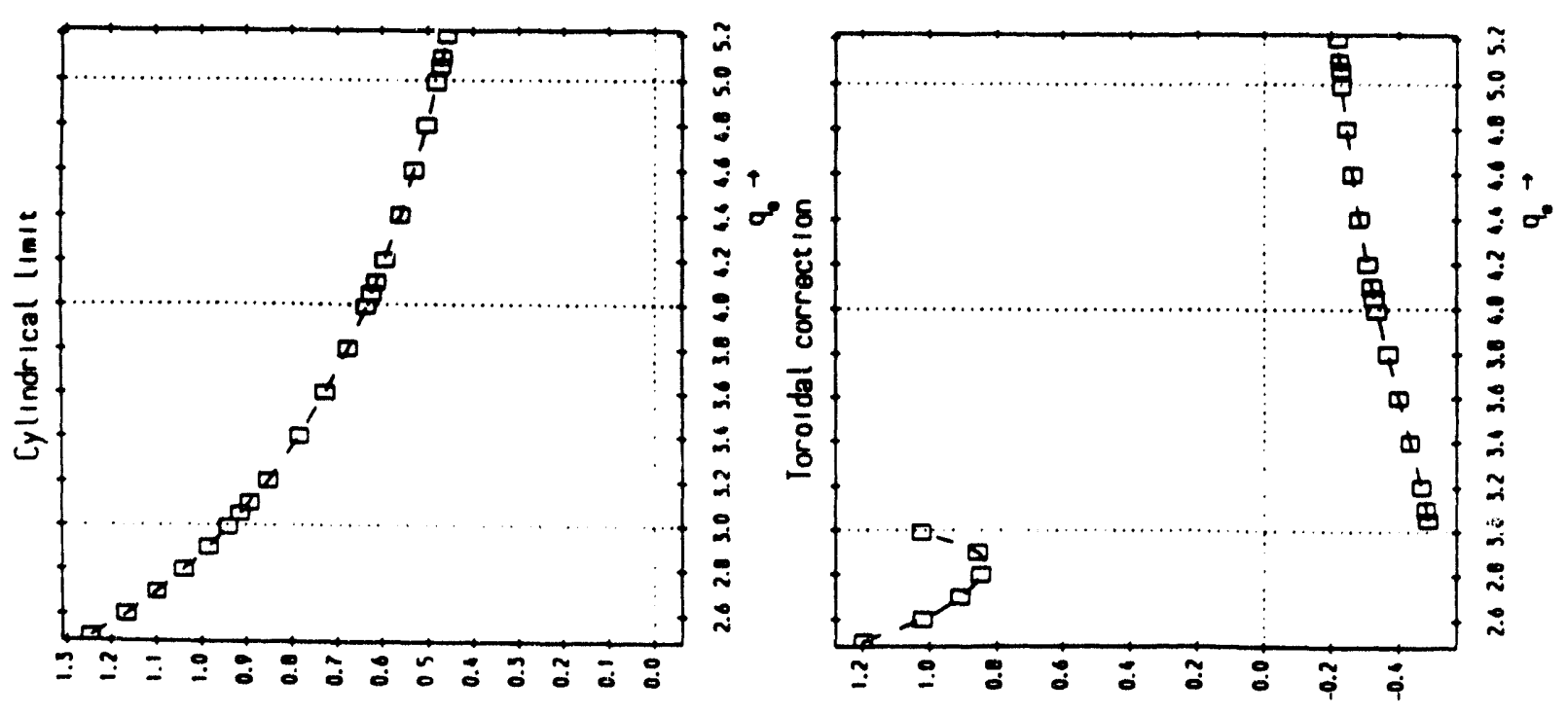


TSH

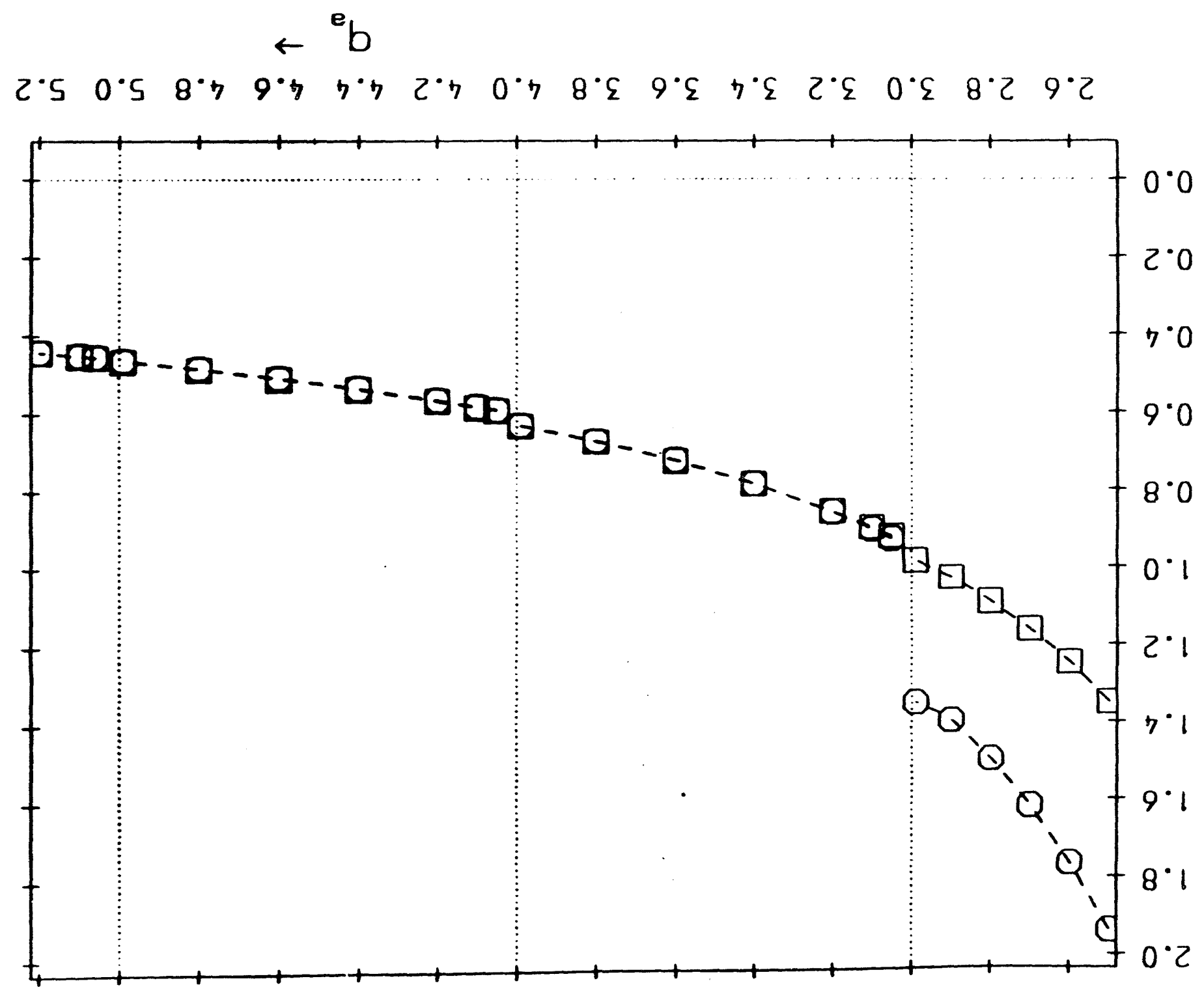




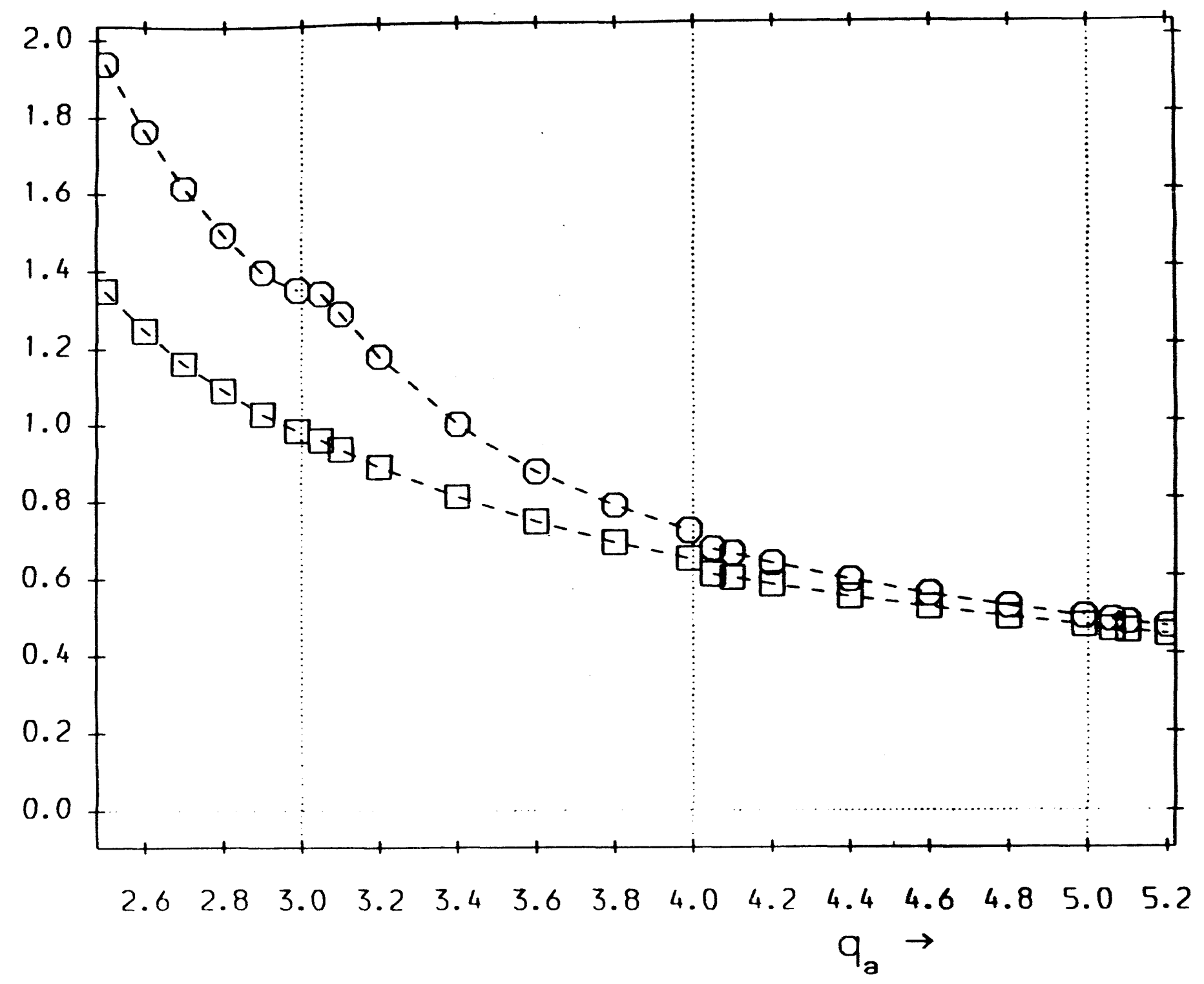

Fis 


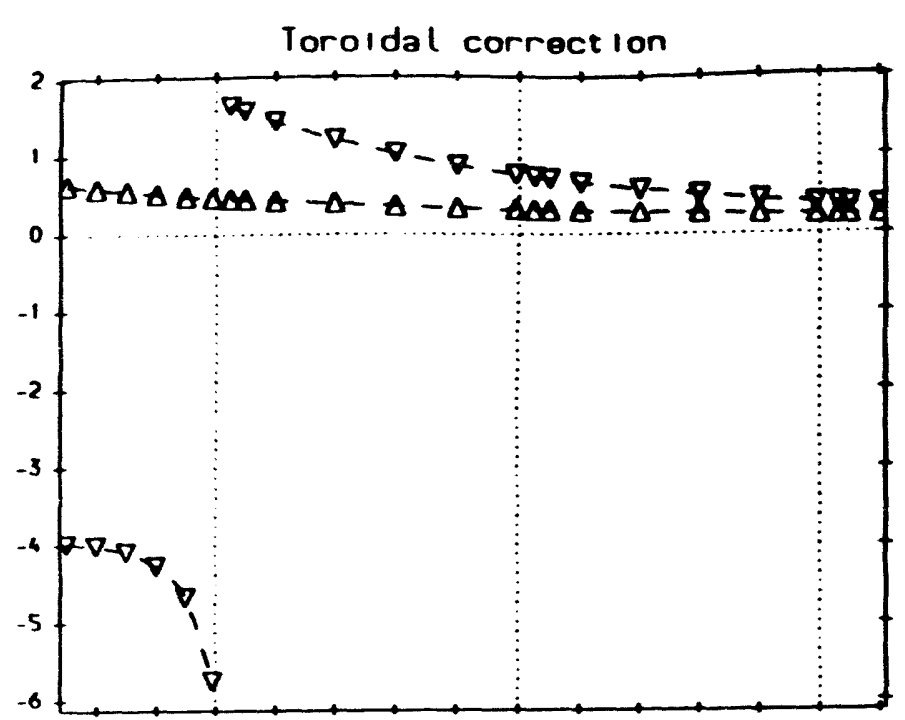

$\begin{array}{llllllllllllllll}2.6 & 2.0 & 3.0 & 3.2 & 3.4 & 3.6 & 3.0 & 4.0 & 4.2 & 4.4 & 4.0 & 0.0 & 5.0 & 5.2\end{array}$

q. $\rightarrow$

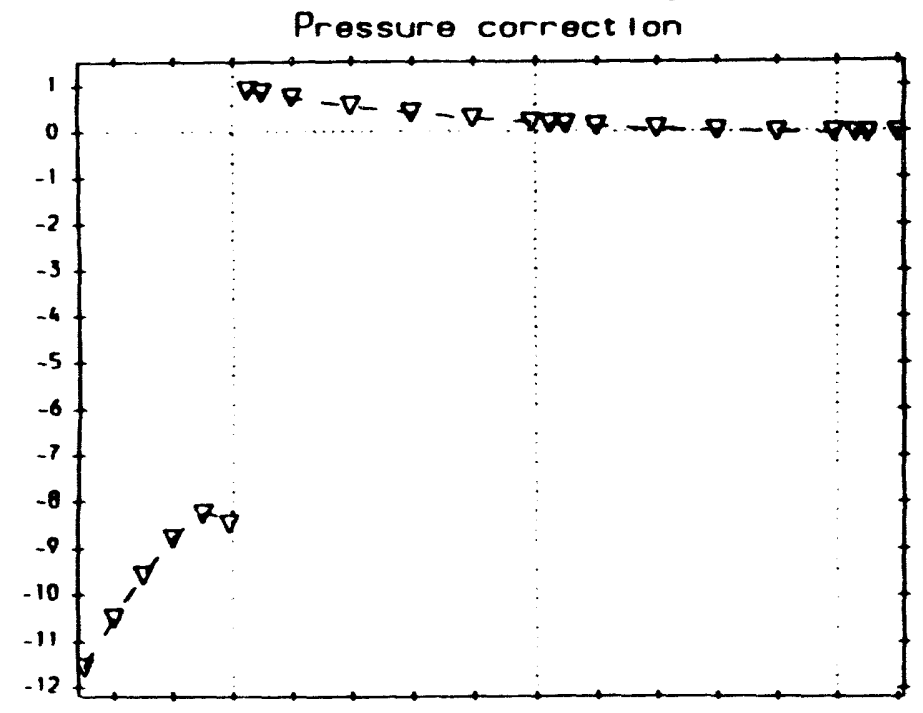

$\begin{array}{llllllllllllllll}2.6 & 2.6 & 3.0 & 3.2 & 3.6 & 3.6 & 3.8 & 4.0 & 4.2 & 4.6 & 4.6 & 4.8 & 5.0 & 5.2\end{array}$

9. $\rightarrow$
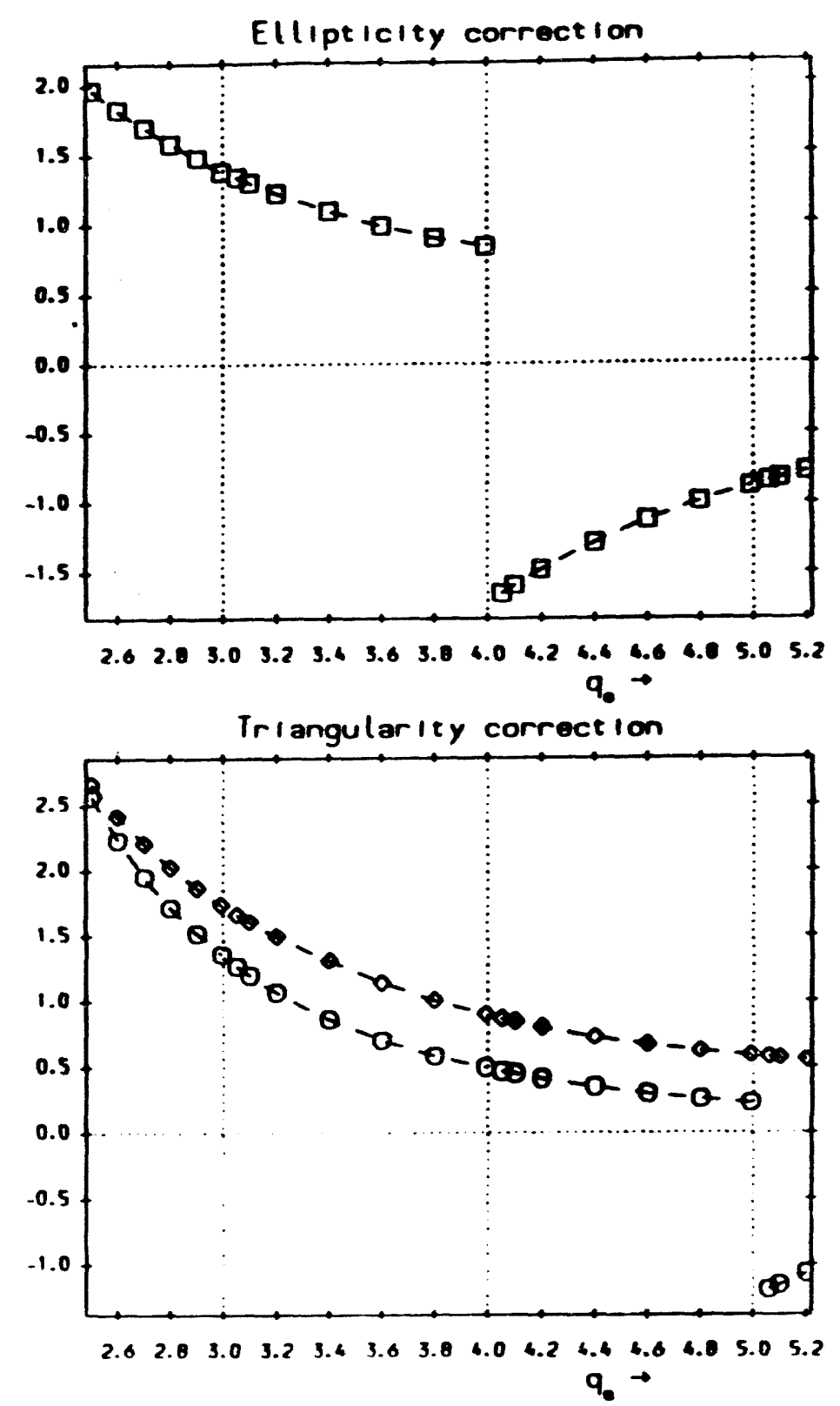

F156 


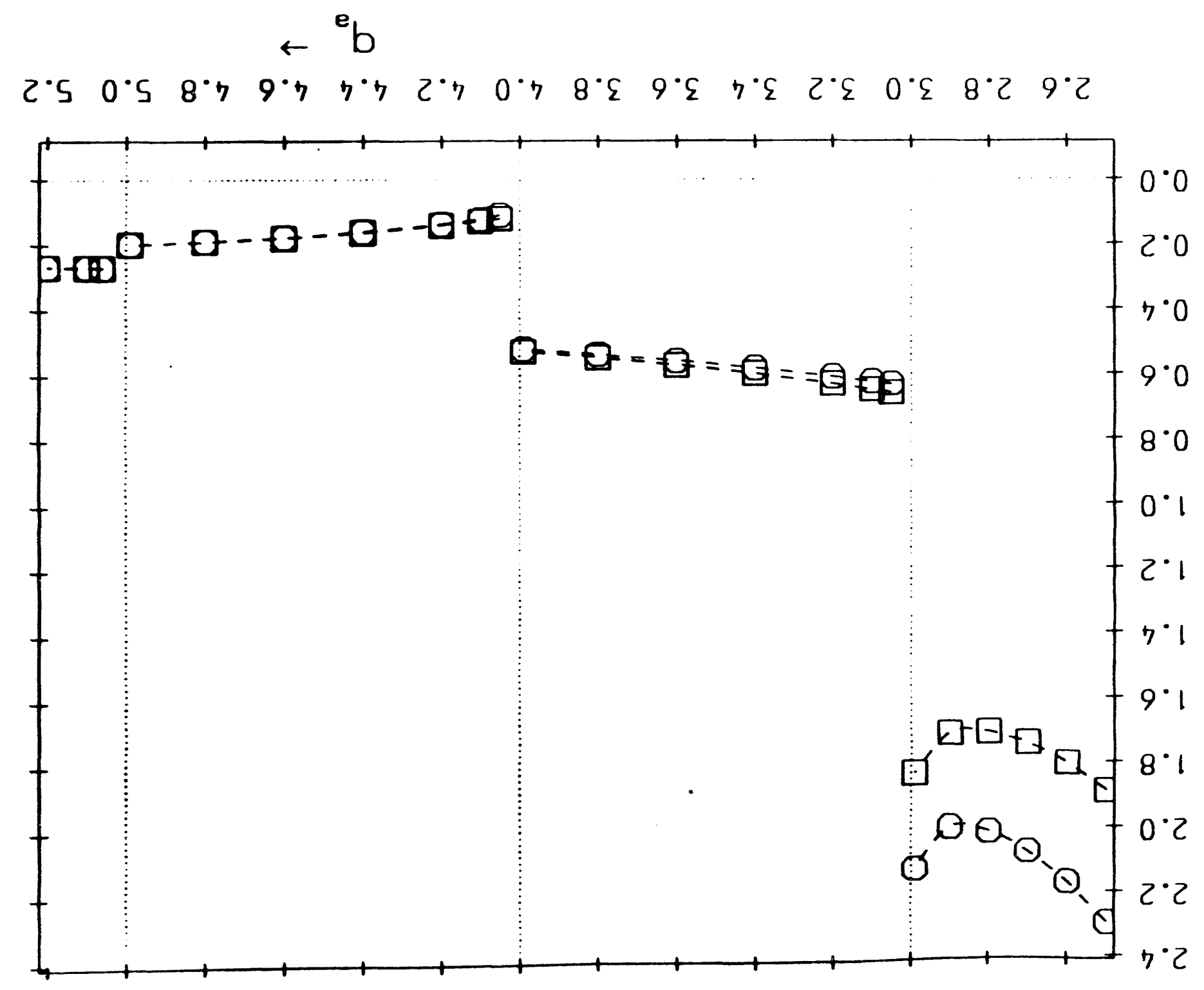




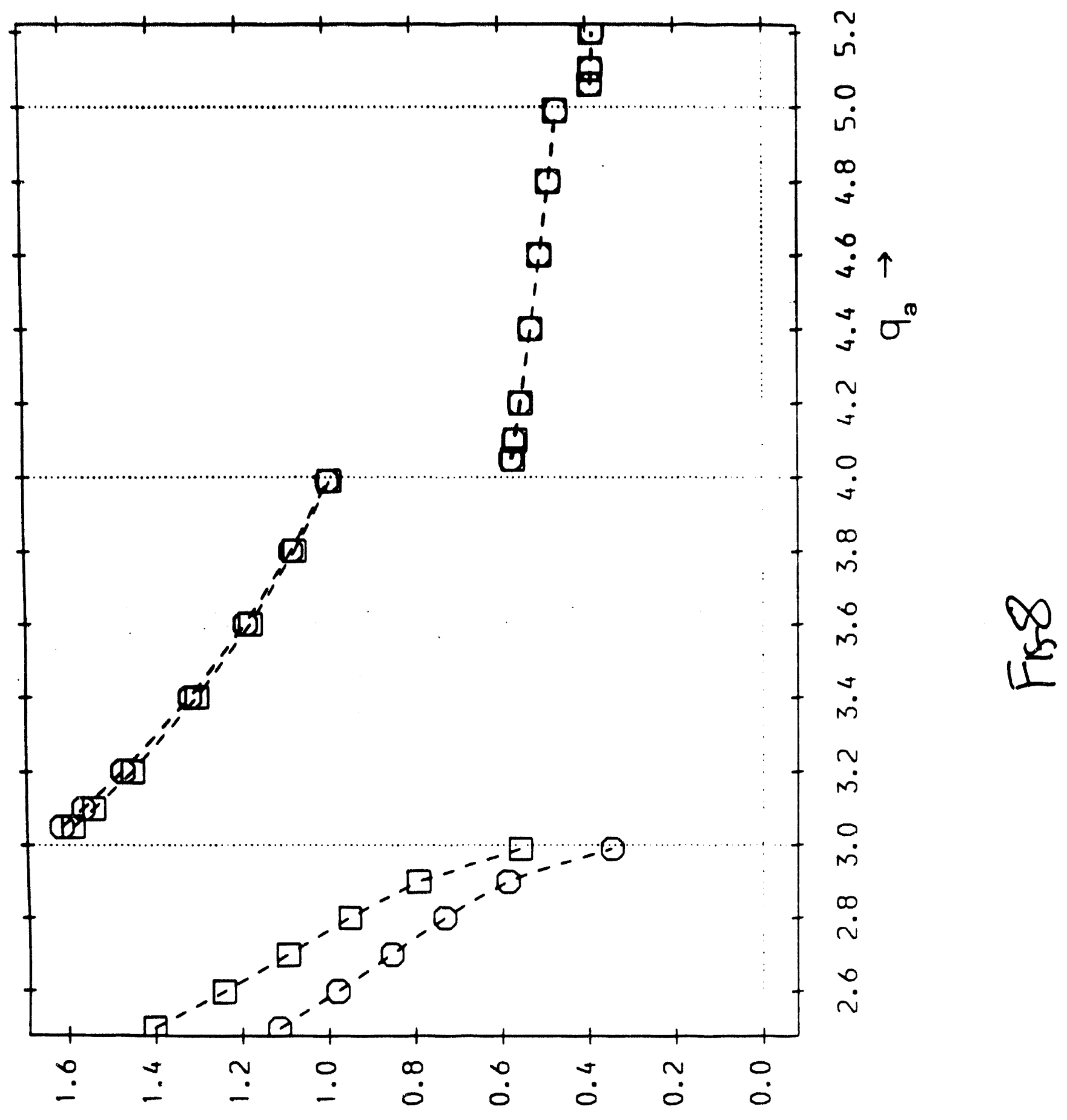




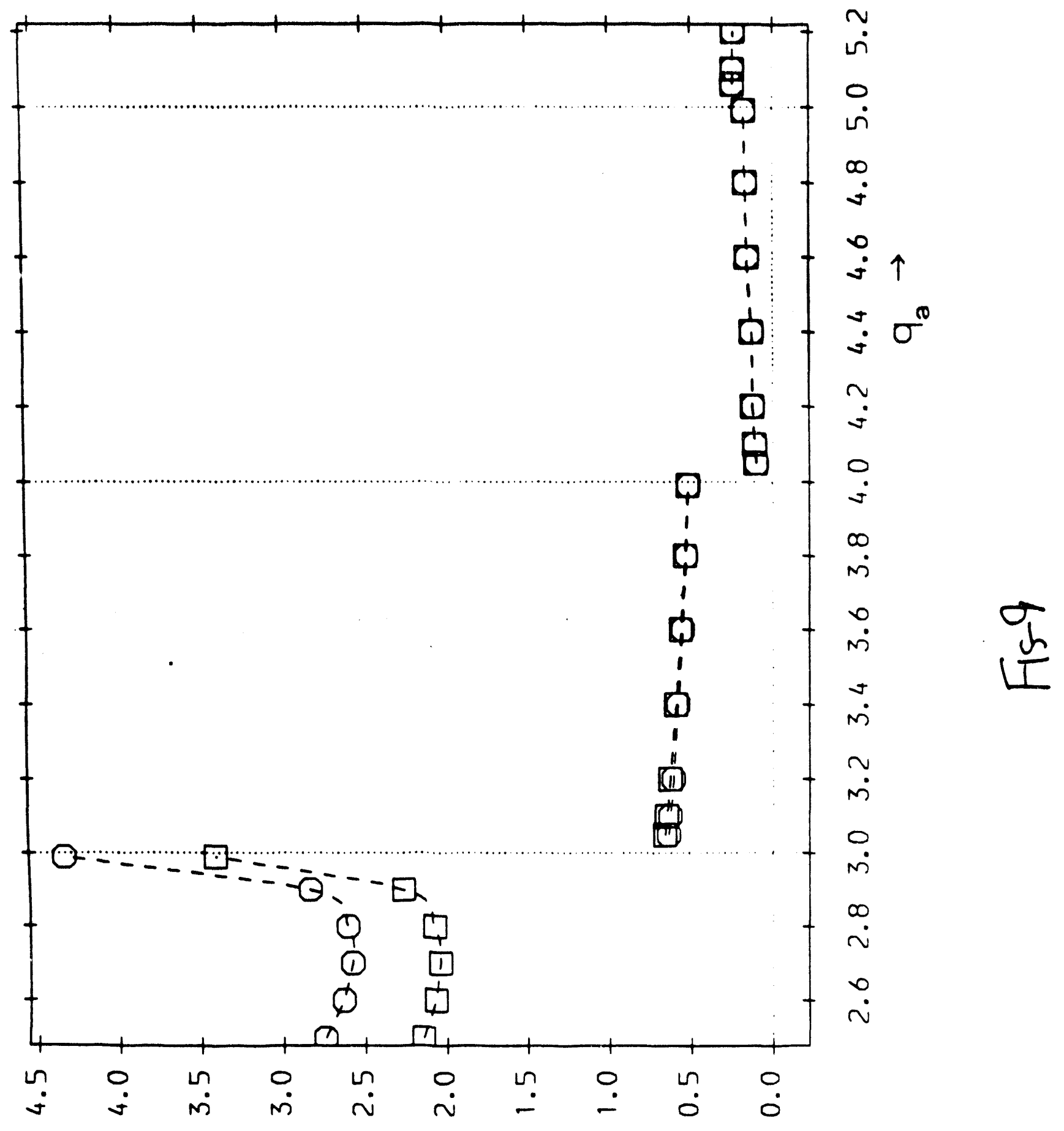



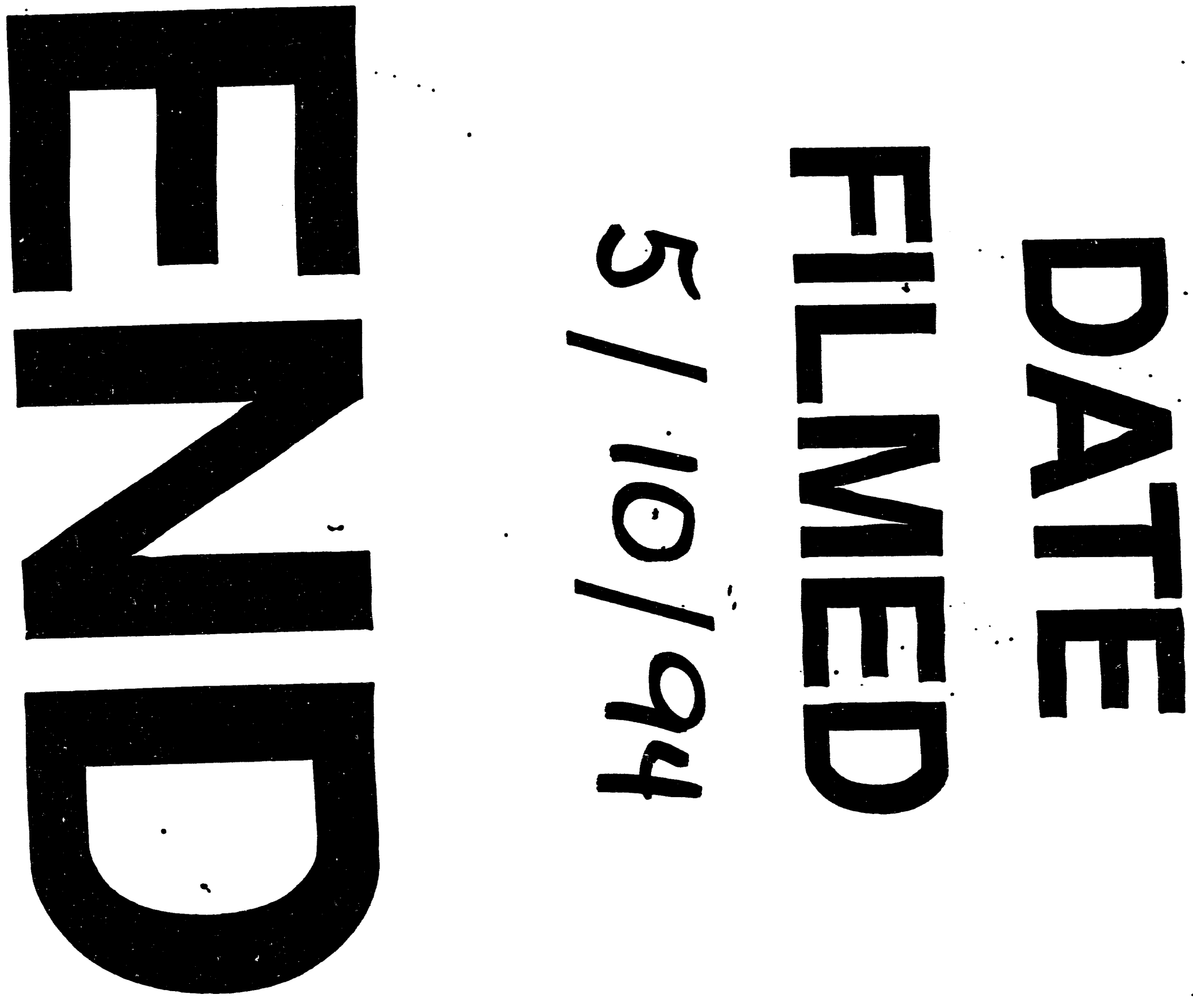
D 01 